\title{
PROCEEDINGS OF THE THIRTIETH ANNUAL MEETING OF THE AMERICAN SOCIETY FOR CLINICAL INVESTIGATION HELD IN ATLANTIC CITY, N. J., MAY 2, 1938
}

\author{
READ BEFORE THE SCIENTIFIC SESSION
}

The Successful Treatment of Pernicious Anemia by Means of Non-Autolyzed Yeast. By Maxwell M. Wintrobe, Baltimore, Md.

It has been the general opinion that yeast, if it possesses any antianemic potency whatever, is effective only after autolysis and then only by virtue of its content of "extrinsic factor." The observations reported contradict this view and indicate that dehydrated yeast which has not been subjected to autolysis, contains an antipernicious anemia substance. Yeast obtained from two different sources was effective in the treatment of classical cases of pernicious anemia. This effect occurred without mixture with gastric juice from normal persons, in amounts as low as 1 gram per kilogram per day. When 2 grams were given per $\mathrm{kgm}$. per day, the results of treatment were as good as those following the oral administration of liver extract derived from 600 grams of liver daily or those following the use of dessicated stomach derived from 265 grams of stomach daily.

The hemopoietic effect was slightly increased by mixture of the yeast with normal gastric juice but only to an extent comparable with the effect of such gastric juice on liver extract. The antianemic effectiveness of yeast was demonstrated in patients who had failed to respond to the administration of beef muscle.

The Coagulation Defect in Hemophilia. The Use of a Globulin Substance Derived from Beef Plasma as a Local Hemostatic in Hemophilia. By Frederick J. PoHle and F. H. L. TAYlor (introduced by George R. Minot), Boston, Mass.

Previous studies by Patek and Taylor 1 and Pohle and Taylor ${ }^{2}$ have suggested that the defect in coagulation of the blood in hemophilia is due to the absence or alteration of a clot accelerating factor associated with a plasma globulin fraction. This globulin fraction prepared by isoelectric precipitation at $\mathrm{pH} 6.0$ from normal human plasma had marked clot accelerating powers for hemophilic blood in vitro and in vivo. Patek and Taylor (1) prepared a globulin fraction in the same way from certain animal plasmas which possessed in vitro activity.

The present observations demonstrate that beef plasma yields a globulin fraction with similar in vitro properties. Its daily oral administration to hemophilics was without effect on the coagulation time of the blood. Parenteral administration has not yet been attempted.

Beef globulin substance was shown to be highly effective as a local hemostatic in hemophilia. When applied

1 Patek, Arthur J., Jr., and Taylor, F. H. L., J. Clin. Invest., 1937, 16, 113.

2 Pohle, Frederick J., and Taylor, F. H. L., J. Clin. Invest., 1937, 16, 741. in powdered form hemostasis was readily obtained in hemorrhages following nine dental extractions and three external wounds in five hemophilic subjects. When applied in liquid form as other hemostatics are usually em1 loyed the results were unsatisfactory. Since the coagulation time of the circulating blood was unchanged the effectiveness of powdered beef globulin substance when locally applied to a bleeding wound in hemophilia is attributed to the rapid formation of a firm fibrin clot. The failure of liquid preparations may be due to the inability to maintain a sufficient concentration of the clot accelerating material at the site of injury during the several minutes necessary for clot formation.

The Experimental Production of Various Types of Hemolytic Syndromes by Hemolytic Sera with Especial Reference to Spherocytosis, Increased Fragility Test, and Reticulocytosis. By William Damesher, and (by invitation) Steven O. Schwartz, Boston, Mass.

In a previous study, hemolysins active against all the blood groups were demonstrated in the sera of 3 cases of acute hemolytic anemia. Marked spherocytosis and greatly increased red cell fragility, present in one of the cases, became diminished with diminution of the hemolytic titer of the serum. On the assumption that these phenomena might be due to the hemolysin present, attempts were made to reproduce them experimentally. Guinea pigs were injected with heterophilic hemolytic rabbit sera produced by sensitizing the rabbits to guinea pig red cells. The various hemolytic syndromes encountered in human pathology, i.e. hemoglobinuria and acute, subacute, and chronic hemolytic anemias were readily reproduced. Spherocytosis, increased erythrocyte fragility, reticulocytosis, and hemoglobinemia and hemoglobinuria could be produced at will depending solely upon the amount and frequency of dosage. On the basis of these and other observations, the conclusion seems justified that spherocytosis and increased fragility of the red cells in hemolytic anemia may be due to the activity of an hemolysin rather than to defective formation of the red cells in the bone marrow. These observations also demonstrate the close physiological relationship between states of acute hemoglobinuria, acute hemolytic anemia (icterus), and subacute and chronic hemolytic anemias.

Further Studies of Antigenic Differences in Strains of the Virus of Epidemic Influenza. Вy Tномаs $\mathrm{P}$. Magill (by invitation), and Thomas Francis, JR.. New York, N. Y.

In earlier studies with the virus of epidemic influenza the results of serological and immunological tests indi- 
cated that all strains of the virus were immunologically identical but that they were readily distinguished from the virus of swine influenza. In 1937 Magill and Francis succeeded in demonstrating that certain antigenic differences of minor degree did exist between strains of epidemic influenza virus.

Since that time further studies have been carried out which have confirmed and extended the original observations. It has been possible to show that certain strains are sharply different from one another and that these differences may be demonstrated not only by cross neutralization tests in mice but by active immunization.

The Time of Appearance and Subsequent Changes in Titer of the Virus Neutralizing Antibodies in the Course of Encephalitis, St. Louis Type. By J. E. Greutter (by invitation), G. O. Broun, and (by invitation) A. E. Casey and R. O. Muether, St. Louis, Mo.

Studies of cases of epidemic encephalitis occurring in the St. Louis area in the summer of 1937 show that virus neutralizing antibodies may be found as early as the third or fifth day after the beginning of prodromal symptoms and may be present on the day of onset of acute symptoms. It would appear that virus neutralizing antibodies develop during the prodromal period. The prodromal symptoms must, therefore, constitute a definite part of the clinical course of the disease. Little evidence of change in titer of virus neutralizing antibodies was found in the same patient even four years after the acute stage of the disease. Different patients vary considerably in the titer of antibody which they develop.

Between twenty and twenty-five per cent of cases showing typical findings of epidemic encephalitis fail to show virus neutralizing antibodies both in the epidemic of 1933 and that of 1937. It is not clear whether these cases fail to produce antibodies or represent individuals infected with a strain of encephalitic virus not yet tested or isolated, or finally whether they are atypical cases of poliomyelitis or some similar infection.

On the Permanence of Recovery in Acute Glomerulonephritis. By EmILY Nichols LoEb and JoHN D. Lyttle (by invitation), David Seegal and (by invitation) Elizabeth L. Jost, New York, N. Y.

1. A clinical, bacteriological, and immunological study with reference to hemolytic streptococcal infection has been made in 10 patients. These individuals were observed (1) during their acute glomerulonephritis, (2) throughout a subsequent healed period, and (3) during and following a subsequent infection with the hemolytic streptococcus.

2. Eight of the patients whose nephritis at onset was preceded by hemolytic streptococcal infection were observed through healed periods varying from 8 months to 3 years. Thereafter, in each instance, an intercurrent hemolytic streptococcal infection produced no recurrence of their nephritis.

3. Two of the patients with acute glomerulonephritis, preceded by hemolytic streptococcal infection in one and by Type I lobar pneumonia in the other, were observed through subsequent healed periods varying from 10 months to 10 years. Each then underwent an infection proven due to the hemolytic streptococcus in one instance and presumably due to that organism in the other. Both developed transient gross hematuria without significant albuminuria.

4. No one of the 10 patients studied has developed the chronic form of glomerulonephritis.

Culture of Human Marrow: A Comparative Study of the Effects of Sulfanilamide and Antipneumococcus Serum on the Course of Experimental Pneumococcus Infections. By EDwIN E. Osgood and (by invitation) Inez E. Brownlee, Portland, Oregon.

The vaccine vial technic of human marrow culture permits quantitative studies of the interaction of living, human cells and noxious and therapeutic agents under accurately controlled conditions, not attainable in animal experimentation or clinical investigation. Such cultures were inoculated with pneumococci of the desired type, mixed thoroughly, and equal volumes transferred to each of a number of vials, one of which was left as a control. To the others, various concentrations of sulfanilamide, antipneumococcus serum or combinations of the two were added. These were mixed and samples removed for bacterial counts and hematologic studies at once and at intervals. Logarithmic growth curves were plotted from the colony counts.

Sulfanilamide alone exhibited a slight bacteriostatic action on pneumococcus infections. Concentrations of $1: 10,000$ were not as effective as antipneumococcus serum alone and did not influence phagocytosis or dissolve capsules. Sulfanilamide plus antiserum was more effective than the corresponding concentrations of either alone. If these results are applicable to human infections, sulfanilamide therapy should be of value in pneumococcus infections but will not prove as effective as antiserum. If used with antiserum, it should lower mortality and decrease expense by decreasing the amount of antiserum needed. Sulfanilamide in combination with therapy designed to introduce or develop specific bactericidins should be investigated further as a method of treatment for infections which are relatively resistant to the action of sulfanilamide alone.

Vicarious Metabolic Response: The Oxygen Consumption of Surviving Tissues in Plasma from Hyperthyroid Organisms. By W. T. SALTER and (by invitation) F. N. Craig, Boston, Mass.

Surviving tissues show no immediate elevation in rate of oxygen consumption when thyroxine is added to the perfusing fluid (Locke's or plasma). Nevertheless, within 4 hours after equilibration with plasma from hyperthyroid guinea pigs and mice, sarcoma tissue shows a distinct elevation of metabolic rate. More striking is the response of pedigreed-mouse liver, which occurs al- 
most immediately-provided experimental conditions are favorable.

The increased basal metabolic rate of the animal is thus reflected vicariously in the metabolic rate of the pedigreed tissue as measured by Warburg's technique. The indirect method obviates such complications as nervous activity, surface-area standards, and possible sub-standard or hyper-standard response of mongrel tissues to a definite concentration of hormone.

A few patients' bloods have been tested: e.g., exophthalmic goiter B.M.R. + 36, + 33, V.M.R. + 33; exophthalmic goiter B.M.R. + 70, V.M.R. +35 ; myxedema under treatment B.M.R. - 21, V.M.R. - 23; cardiac decompensation B.M.R. + 30(?), V.M.R. 0. Myeloid leukemia B.M.R. + 36, V.M.R. + 5. "Nephrosis" treated with heavy doses of thyroxine intravenously B.M.R. - 21, V.M.R. - 6. Of 11 human cases of frank thyroid dysfunction, 8 fell very close to a straight line when V.M.R. was plotted against B.M.R. In 4 hyperthyroid cases V.M.R. fell promptly with iodine therapy. Five cases of myeloid leukemia with B.M.R. ranging from +28 to +56 all gave V.M.R. close to 0 . Evidently the V.M.R. has special interest in hypo- and hyperthyroidism.

The technique at present is not sensitive enough to distinguish mild degrees of hyperthyroidism, but better quantitative adaptation of the method is being attempted.

The Urinary Excretion of Prolan, Estrin, and Pregnanediol in Normal Pregnancy and in Early and Late Pregnancy Toxemias. By J. S. L. Browne and (by invitation) J. S. Henry, and Eleanor H. Venning, Montreal, Canada.

The excretion of these substances in 24 hour specimens of urine has been studied in eight normal cases, four cases of pernicious vomiting of early pregnancy and twenty-one cases of late pregnancy toxemia. In some of these cases the determinations have been done over long periods of time. Prolan was determined by benzoic acid precipitation and assay on the immature rat, estrin by the colorimetric method of Venning et al., and pregnanediol (which is regarded as an excretion product of progesterone) gravimetrically as sodium pregnanediol glucuronidate. Time in pregnancy is expressed in days after the beginning of the last menstrual period.

The existence of a peak of prolan excretion at 50 to 60 days and a subsequent decline to a low level by about 120 days is confirmed. In some entirely normal cases there is a second rise of prolan excretion occurring during the seventh month and lasting in some cases for $1 \frac{1}{2}$ to 2 months, declining in the ninth month. Most of the late pregnancy toxemic cases showed values for prolan which were within the normal range, tending to be low rather than high.

Estrin is excreted almost entirely in the conjugated form throughout pregnancy. The rate of excretion increased from about 100 to 200 gamma per 24 hours at 40 days to 1000 to 2000 gamma at 80 to 110 days. At about 110 days there was a definite turn upward in the curve of estrin excretion. The rate rose rapidly thereafter reaching 10,000 to 15,000 gamma at about the 150th day. In some cases the rise was interrupted by slight decreases which tended to occur at regular intervals. In those cases showing the second rise of prolan mentioned above, the curve of estrin excretion also showed a second sharp upward rise, increasing in one case from 15,000 gamma on day 168 to 40,000 gamma on day 250 . The highest point of estrin excretion varied from 15,000 to 40,000 gamma and was reached during the ninth month; this value is for normal cases. The estrin excretion in cases of late toxemia was in most cases lower than nor$\mathrm{mal}$; but there were exceptions.

The pregnanediol excretion in the normal cases in general paralleled that of the estrin. The values for normal pregnancy have been reported previously. The pregnanediol excretion in most of the late pregnancy toxemias was definitely lower than normal, for example in one severe case it was $7.3 \mathrm{mgm}$. per 24 hours at 250 days as opposed to a normal value of 60 to $80 \mathrm{mgm}$. In none of the cases studied has it been completely absent.

Definite correlation of the level of excretion of either prolan, estrin or pregnanediol with the degree of toxemia was not possible.

In one case of early pernicious vomiting the prolan excretion was 300 R.U. at 72 days which is low, in the other cases it was within normal limits. In all cases the estrin and pregnanediol were within normal limits.

The hypothesis is advanced that the low values for estrin and pregnanediol found in late toxemias do not necessarily result from a decreased production of these substances but may equally well be due to their retention in the body from failure of their conjugation with glucuronic acid (which probably takes place in the liver) and of their excretion by the kidney. Estrin is known to cause water retention in animals and human beings and to be highly toxic to adrenalectomized animals. It is suggested that some of the symptoms of late pregnancy toxemias may be contributed to by intoxication with retained estrin.

The Effect of Testosterone Propionate on the Genitalia, Prostate Secondary Sex Characters, Nitrogen, Salt and Energy Metabolism in Eunuchoidism and in Eunuchoidism of Pituitary Origin. By ALLAN T. KENyon, Irene Sandiford, A. Hughes Bryan, Kathryn KNowston and F. C. KocH (introduced by O. $\mathrm{H}$. Robertson), Chicago, Ill.

Five eunuchoids received $25 \mathrm{mgm}$. of testosterone propionate from three to seven times weekly for from three to five months. Increased frequency of erections, enlargement of the penis (in four) and of the prostate, deepening of the voice (in four), breast hypertrophy (in one) and increase in sexual hair was produced together with a marked gain in body weight. Three eunuchoids treated in the hospital while on constant diet and regulated activity for from 10 to 15 days showed a retention of nitrogen of from 23 to $63 \mathrm{grams}$, without change in the nitrogenous blood constituents, an irregular depres- 
sion of urinary creatine, a retention of from 3.5 to 7.7 grams of sodium, a gain in weight of from 1.3 to 3.5 $\mathrm{kgm}$. largely due to water associated with salt and protein, and a slight increase in basal metabolic rate. A eunuchoid with a suprasellar cyst showed similar changes, together with enlargement of the prostate, except that there was no rise in basal metabolism.

Biochemical and Clinical Studies of Virilism Before and After Removal of Adrenal Cortical Tumor. By H. B. FrIEDGOod (by invitation), and S. L. GARGILL, Boston, Mass.

A white girl, aged 10 , with a malignant adrenal cortical tumor showed hirsutism, masculine voice and muscular development, acne, hypertension, polycythemia, acrocyanosis, hypertrophied clitoris, purplish striae, and menstruation for 6 months at the age of 8 . Complete biochemical and clinical studies were made before and after adrenalectomy. Eight consecutive 24-hour urine speciments collected before operation and 10 collected at intervals postoperatively were assayed for androgens (using the modified Koch method of extraction and purification) by Oesting's colorimetric method, based on Zimmerman's color reaction of sex hormones. Androgen excretion ranged from 625 to 842 I.U. per 24 hours, preoperatively. During 24 hours succeeding operation 120 I.U. were excreted. Values fluctuated between 9 and 51 I.U. during the following 3 months. Normal androgen excretion for this age ranges from 1 to 10 I.U. per day. A normal adult female excretes about 40 to 50 I.U. per day. Estrogen excretion is also being determined. The tumor was also assayed for androgens. The benzene-soluble fraction, in which the androgens are ordinarily found, contained 8.5 I.U.

The mild hypertension and polycythemia disappeared almost immediately after operation. The hirsutism, acne, and hypertrophied clitoris have regressed considerably since operation. Catamenia reappeared one month after operation and has returned periodically every 30 days. At present her androgen excretion equals that of a normally menstruating adult female. Determinations are being made frequently to detect early evidence of metastatic activity. Photographs of the patient are available before and after operation.

The Mechanism of Vitamin C Excretion in Man Studied by Simultaneous Vitamin $C$ and Inulin Clearances. By Elaine P. Ralit, Gerald J. Friedman and Saul H. RuBIN (introduced by J. Murray Steele), New York, N. Y.

Simultaneous vitamin $\mathrm{C}$ and inulin clearances were done by the constant infusion method described by Smith, Goldring, and Chasis. The 2, 6 dichlorophenol-indophenol titration method was used for the determination of vita$\min C$ in plasma and urine.

The vitamin $\mathrm{C} /$ inulin clearance has a ratio of less than 0.02 at plasma levels of the vitamin below $2 \mathrm{mgm}$. per cent. As the plasma level of the vitamin is elevated the clearance ratio rises and approaches 1.0. Analysis of the respective rates of filtration and excretion of the vitamin show that at all plasma levels above $2.0 \mathrm{mgm}$. per cent the renal tubules reabsorb a constant maximal quantity of the vitamin per minute. In two normal individuals this quantity was 2.84 and $2.48 \mathrm{mgm}$. per cent.

The renal "threshold" of vitamin C appears therefore to be due to the fact that the renal tubules can almost completely reabsorb this substance from the glomerular filtrate, up to a maximal rate which is presumably constant in each individual. In this respect the vitamin threshold resembles the glucose threshold as recently described by Shannon, in that it is not an independent physiological entity but is the resultant of two variables that may be capable of independent variation.

Since vitamin C is excreted solely by glomerular filtration it follows that the rate of excretion at a given level will be reduced pari passu with reduction in filtration rate when the latter is reduced by disease.

The Rôle of Nicotinic Acid in the Prevention of Pellagra, Roentgen Sickness, and Increased Porphyrinuria. By Tom D. SpIEs and (by invitation) William B. Bean, Cincinnati, Ohio.

Within recent months it has become clear that nicotinic acid is a useful therapeutic agent in the treatment of pellagra. The present study is concerned with the prevention of recurrences of the manifestations of pellagra in eleven persons. Increased porphyrinuria is one of the integral parts of the pellagra syndrome and can be prevented by means of nicotinic acid. This observation led us to study the increased porphyrinuria associated with roentgen sickness, lead poisoning, barbital intoxication, anemia, cirrhosis of the liver, and various infections in fifty patients. In every instance the increased excretion of porphyrin returned to normal following the administration of nicotinic acid. The porphyrinuria associated with these conditions, like that associated with pellagra, has been shown in this study to be preventable by means of nicotinic acid. The mode of action of nicotinic acid in the pathogenesis of pellagra, roentgen sickness, and the increased porphyrinuria associated with the other diseases mentioned in this study was discussed.

\section{A Study of the Electrolyte Physiology in a Case of Fa-} milial Periodic Paralysis. By Joseph W. Ferrebee (by invitation), and DANA W. Atchley and RoBerT F. LOEB, New York, N. Y.

A classical case of familial periodic paralysis occurring in a 30-year old single male has been studied on the metabolism wards of the Presbyterian Hospital for the past six months. Dietary intakes have been standardized and duplicate diets analyzed in $\mathbf{5}$ day periods for sodium, potassium, chloride, calcium, nitrogen, and water contents. All urines and stools have been similarly treated. Total plasma volumes, total extracellular fluid volumes, hematocrits, and chemical analyses of the blood serum (sodium, potassium, calcium, chloride, phosphate, carbonate, glucose, albumin, and globulin) have been done at significant intervals. From time to time the sodium and 
potassium intakes have been altered and the resulting changes, both immediate and delayed, have been observed.

The results have been a confirmation of the effectiveness of potassium chloride by mouth in alleviating and preventing attacks of paralysis.

The existence of a low serum potassium during attacks has been confirmed. No correlation between this low serum potassium and the serum glucose or phosphate levels has been observed.

A marked drop in urinary potassium excretion, independent of urinary volume, has been found to occur during an attack; and following an attack a compensating increase in potassium excretion has been observed.

A constant creatinuria has been found to increase as much as $\mathbf{5 0}$ per cent during an attack.

The acetylcholine esterase activity of the serum of this patient has been found to be less than that of controls.

Renal Function in Experimental Adrenal Insufficiency. By Harold E. Harrison (by invitation) and DaNiel C. Darrow, New Haven, Conn.

The renal clearances of creatinine, urea, potassium, sodium, chloride, and phosphate were determined in an intact dog, and in the same animal following bilateral adrenalectomy during periods of adrenal insufficiency and subsequent to adequate therapy with sodium salts and adrenal cortical extract. The creatinine clearance was taken as an index of the volume of glomerular filtrate.

During adrenal insufficiency the creatinine clearance dropped to about 25 per cent of the normal level. The urea clearance roughly paralleled the creatinine clearance indicating that urea retention in adrenal insufficiency is essentially due to diminished glomerular filtration. Both the creatinine and urea clearances were rapidly restored to more nearly normal levels by the administration of a hypertonic solution of sodium chloride and sodium bicarbonate.

The administration of potassium salts to a normal dog results in the excretion of potassium in the urine at a maximum concentration of approximately 40 times the serum concentration irrespective of the urine volume, within wide limits. Following adrenalectomy the maximum concentration of potassium in the urine is only about 10 times the serum concentration. The administration of sodium salts to the adrenalectomized dog does not increase the ability of the kidneys to excrete potassium at a high concentration but because of greater urine volume more potassium may be excreted per hour. Within a few hours after the injection of cortical hormone the ability of the kidneys to maintain a high concentration of potassium in the urine is restored.

Effective Renal Blood Flow and Functional Excretory Mass in Essential Hypertension. By William Goldring, Herbert Chasis, Hilmert A. Ranges, and $\mathrm{H}$. W. Smith (introduced by Dr. William Tillett), New York, N. Y.

Twenty-one subjects with essential hypertension have been examined in respect to renal blood flow and filtra- tion rate, many of them on two or more occasions. In fourteen subjects the functional excretory mass, $T_{m}$, as measured by the maximal rate of excretion of diodrast, has been determined. These subjects consistently show functional destruction of renal tissue, $T_{m}$ being reduced in advanced disease to less than 10 per cent of normal. They also all show a renal blood flow less than normal (ca. 1360 cc. per minute), ranging as low as 60 cc. per minute. With few exceptions the renal blood flow per unit of excretory mass is below the normal range, indicating relative ischemia of the residual functional tissue.

In every case the fraction of plasma filtered at the glomerulus (as calculated from the inulin clearance and the effective renal plasma flow) is greater than normal. This circumstance might be due to (1) the persistence of tubules which have lost their excretory capacity, but which retain connection with their glomeruli and serve as conduits for glomerular filtrate; (2) increased glomerular pressure associated with the existing hypertension, or (3) hypertonus of the efferent glomerular arterioles. Final selection between these possibilities requires further experimental exploration of the functional contribution of (2) and (3), but the available data suggest that efferent arteriolar spasm is an important factor in the induction of both the increased filtration fraction and the relative ischemia of the residual functional tissue.

Our observations may be interpreted as in accordance with those of Goldblatt and his coworkers and of others who have shown that renal ischemia can induce hypertension in dogs, but the conclusion that renal ischemia is a causal factor in hypertension in man cannot be reached until it is demonstrated that the observed ischemia is not simply a concomitant of generalized systemic hypertonus.

Methods for the measurement of renal blood flow and excretory mass will be discussed briefly.

Studies of the Blood Pressure and Circulation by Perfusion of Cadavers with and without Previous Vascular Disease. By JoHn Russell Smith (by invitation) and William Bryan Kountz, St. Louis, Mo.

Increased and peripheral resistance has long been considered a fundamental cause of hypertension. Recent studies have led many to believe that muscular spasm of the finer blood vessels may be important in producing and maintaining hypertension. Pathological findings indicate, however, that hypertension is probably due to factors other than spasm alone.

In an attempt to evaluate the importance of the various factors in hypertension, cadavers taken immediately after death were perfused by an artificial heart, the rate and stroke volume of which could be maintained constant at widely varying levels. The entire body was perfused as a unit through the aorta with fresh whole animal blood. The influence of changing cardiac rate, or stroke volume, influence of the character of the perfusate, and the action of drugs on the blood pressure were noted. Correlation between direct arterial pressure with cannulae in place and the cuff blood pressure was studied. Such experi- 
ments were conducted both on cadavers of dogs and on human cadavers, with and without previous vascular disease.

Since it is impossible to study the heart output directly in man, we thought it worth while to attempt to evaluate the influence of the heart in the production of high blood pressure with and without constriction of the arterial system. Many interesting relationships were discovered. Among them was the demonstration that a constant normal range of blood pressure can be maintained with widely varying degrees of peripheral resistance or constriction by changing the output of the heart. When the minute output of the heart is maintained at a constant level, the blood pressure varies directly with peripheral resistance. When heart output per minute and the peripheral resistance remain constant, increase in viscosity of the blood increases the blood pressure. The action of drugs was found to vary considerably depending, not alone upon their known action, but also upon the state of the vascular system. Thus nitrite in extreme states of vascular constriction may elevate the peripheral blood pressure, while pitressin may cause a definite fall. These experiments suggest the idea that one factor in maintaining hypertension may be the size and state of the larger vessels as well as constriction in the smaller ones.

Blood Flow and Vasomotor Reactions in the Foot in Health, in Arteriosclerosis, and in Arterial Hypertension. By Eugene A. Stead, Jr., and Paul Kunkel (introduced by Soma Weiss), Boston, Mass.

Plethysmograph for measuring the blood flow in the foot has been devised on the principle of Hewlett and van Zwaluwenburg. The accuracy of the method has been studied by means of a constant injection apparatus. The measurements of the blood flow were made at $43^{\circ} \mathrm{C}$. ("maximal dilatation") and were expressed in cubic centimeters of blood per $100 \mathrm{cc}$. of foot per minute. The average blood flow per minute in 21 normal males between the ages of 19 and 67 years was $15.8 \mathrm{cc}$; the highest value was 21.6 and the lowest $11.1 \mathrm{cc}$. The average flow in 11 normal females between the ages of 17 and 50 years was 17.6 ; the highest value was 25.2 and the lowest $13.4 \mathrm{cc}$. In the presence of a normal cardiovascular system no appreciable change in blood flow occurred in subjects up to the age of 70 years. In 11 normal subjects measurements of the blood flow made simultaneously in both feet showed an average difference of $2.2 \mathrm{cc}$. In 12 normal subjects simultaneous measurements were made in one hand and one foot. The average flows were, respectively, 32.2 and $15.5 \mathrm{cc}$. These measurements indicate that the foot has a considerably smaller reserve supply of blood than the hand. Thus, in the presence of an equal degree of vascular damage manifestations of ischemia will develop earlier in the foot.

Eight patients with arteriosclerosis of the vessels of the foot with an average age of 63 years, who had either signs or symptoms of deficient local circulation, had an average foot flow of $4.9 \mathrm{cc}$. Six patients, with an average age of 70, who showed clinical evidence of arterio- sclerosis of the foot but who did not have symptoms on moderate exertion, had an average blood flow of $8.3 \mathrm{cc}$. These measurements indicate that the normal "maximal flow" in the feet can become reduced 50 per cent without symptoms and without trophic disturbances. In the presence of dilated vessels, blood flow below a level of $5 \mathrm{cc}$. is often associated with local symptoms and tissue changes.

In 6 patients with arterial hypertension, whose average age was 40 years and whose blood pressure averaged $206 / 130 \mathrm{~mm}$. $\mathrm{Hg}$, the blood flow was essentially normal, both at body temperature and when the vessels were fully dilated by local heat.

In 6 cases of Buerger's disease with an average age of 51 years, who showed intermittent claudication of the calves without specific foot complaints, the average flow was $9.6 \mathrm{cc}$. In 2 cases with both intermittent claudication in the calves and trophic changes in the toes the average flow was $5.3 \mathrm{cc}$.

The vasomotor reactions in the foot are similar to those in the hand. The vessels respond to the same stimuli, namely, temperature, pinch, noise, and psychic changes. The vasodilator response of the feet, however, is slower on warming the body. On direct heating the vessels of both the hands and the feet dilate completely in about 30 minutes. While local heat $\left(43^{\circ} \mathrm{C}\right.$.) usually induces maximal vascular dilatation, immersion of the hands in hot water produces only partial relaxation in the feet. The degree of vasoconstriction induced by cold water $\left(17^{\circ}\right.$ C. $)$ is not influenced by an environmental temperature sufficiently elevated to produce sweating. In turn, the body cannot be cooled by keeping the feet in cold water because of the very slow flow from the feet at low temperatures.

The Estimation of Cardiac Output, and the Determination of Cardiac Abnormalities from a Record of the Heart's Recoil and the Blood's Impacts (Ballistocardiogram). By ISAAC StARR and (by invitation) A. J. Rawson, H. A. Schroeder and N. R. Joseph, Philadelphia, $\mathrm{Pa}$.

Our apparatus is a modification of that of $\mathrm{Y}$. Henderson (1904). The subject lies on a bed suspended from the ceiling and braced to prevent all motion except in the longitudinal direction. Motion in this direction, opposed by a strong spring, is magnified about $\mathbf{5 0 0}$ times by an optical system and photographed.

Normal standards have been set up from records of over 200 normal persons.

In certain pathological conditions the differences from the normal records are very striking. Most cases showing the common electrocardiographic abnormalities are easily identified by characteristic changes of ballistocardiogram. Other cases show abnormalities having no counterpart on the electrocardiogram.

Cardiac output may be calculated from the ballistocardiogram from the following formula-

(Cardiac output $)^{2}=$

$$
\left(419 I_{\text {cor. }}+277 J_{\text {cor. }}\right) A C(0.4 \sqrt{C}-0.05)
$$


when $I$ and $J$ are the height of the two principal ballistocardiogram waves in mm., each corrected (for lack of critical damping) by subtracting 40 per cent of the height of the preceding wave.

$A$ is the aortic cross section area in sq. $\mathrm{cm}$. It may be derived from the patient's age and surface area according to Bazett's formula (1935).

$C$ is the duration of the cardiac cycle, obtained from the heart rate.

The last term is an estimate of the duration of ejection.

In 30 consecutive cases duplicate estimations of cardiac output by the improved ethyl iodide method (Starr and Gamble, 1928) and from the ballistocardiogram were carried out. If one aberrant result, obtained on a nervous patient be omitted, whenever the ballistocardiogram has a normal form there is no significant statistical difference between results obtained by the two methods. When the ballistocardiogram has an abnormal form the estimate of cardiac output by the formula is, in certain cases, lower than the result obtained by ethyl iodide.

The ballistocardiogram makes a new kind of information easily available to clinicians in the routine examination of patients with cardiac and circulatory disease.

\section{$A$ Practical Method of Visualization of the Chambers of} the Heart, the Pulmonary Circulation, and the Great Blood Vessels in Man. By George P. RoBb and Israel Steinberg (introduced by Arthur C. DeGraff), New York, N. Y.

Pulmonary arteriography was first performed by Professor Carvalho and Dr. Moniz of Portugal in 1931. Their method then and now depends upon the injection of a concentrated solution of sodium iodide, which is opaque to $x$-ray, into the heart through a catheter. The difficult nature of the procedure has precluded its general use.

Dr. Werner Forsstman of Berlin conceived of the visualization of the chambers of the heart and in 1931 attempted this in man without success. Today there is no practical method of visualization of the heart, the lungs or the great blood vessels.

Injection of the contrast substance intravenously has been tried in the past and rejected. Despite these failures, the fact that the brilliant vital red dye passes through the heart and lungs in high concentration in the dye cardiac output method, led us to believe that an adequate concentration of opaque substance could be obtained there by means of a similar method of peripheral intravenous injection. Preliminary studies in rabbits supported this thesis and showed that visualization could be secured by the use of organic iodine compounds in amounts comparable to those which might be safely used in man. Clinical trial demonstrated the practicability of the method and led to the selection of diodrast, an organic iodide compound having the chemical name of 3,5 , diiodo-pyridon acetic acid diethanolamine. By rapid intravenous injection we have been able to outline the superior vena cava, the chambers of the heart, the ventricular wall, and the size and shape of the aorta.
Our method consists of the injection of 20 to $45 \mathrm{cc}$. of a 70 per cent aqueous solution of diodrast into the cephalic or basilic vein of the elevated arm through a 12 or 13 gauge needle. The injection must be made in two seconds or less with the patient sitting in position for radiography. Films taken 3 to 5 seconds after the beginning of injection usually show excellent filling of the right auricle, the right ventricle, and the pulmonary arterial tree. The interval to reach the left chambers varies from 6 to 16 seconds and is accurately indicated by the cyanide circulation time.

We have made 215 injections in 123 subjects without serious consequence. There is usually a transient sensation of intense heat shortly after injection accompanied by a moderate fall in the blood pressure and elevation of the heart rate; occasionally by dizziness and nausea; rarely by vomiting and collapse. Seven patients have developed urticaria, 2 angioneurotic edema, and 1 dermatitis medicamentosa ; and in 24 instances a painless thrombophlebitis limited to the site of injection has occurred. Examination of the blood and urine and functional tests of the kidneys and liver before and after injection have revealed no evidence of injury. Studies of the circulation, including electrocardiograms, arterial and venous blood pressure determinations, respiratory tracings and clinical observations, during and after administration, have shown no serious disturbance, even in the presence of heart or lung disease. Physiological and pharmacological evidence suggest that fall in blood pressure is due primarily to the direct dilating effect of diodrast on the arterioles, and that the tachycardia results from reflexes initiated in the brain, the arterioles and capillaries and in certain vasosensory areas, as well as from direct action on the sinoauricular node.

Roentgen Kymographic Studies in Myocardial Infarction.

By J. Hamilton Crawford and (by invitation) RichARd Gubner, Brooklyn, N. Y.

Forty-five cases of myocardial infarction were studied with the multiple slit, moving film, kymograph. Exposures were made in the postero-anterior, and in two left oblique positions, thus visualizing the movements of the anterior and posterior walls of the left ventricle.

The changes observed varied from reversal of movement to absent pulsation in the infarcted area and corresponded to those observed by Tennant and Wiggers with the myocardiograph in experimental myocardial infarction.

Of the 45 cases, 41 showed definite kymographic changes. In 25 the electrocardiographic and kymographic localization corresponded. In 6 the kymogram indicated involvement of both anterior and posterior walls whereas the electrocardiogram suggested infarction of one or the other. In these the electrocardiogram was probably dominated by the major area of involvement. In 3 cases in which no electrocardiographic localization was possible, due to bundle branch block, the kymogram showed an area of infarction. Six cases of coronary artery disease without definite clinical or electrocardiographic evidence 
of coronary thrombosis showed kymographic changes suggestive of an area of infarction. These patients suffered from either long standing angina pectoris or cardiac decompensation and may have had old infarcts.

Absent or markedly diminished movement especially at the apex must be interpreted with caution in greatly enlarged hearts as it may be present without infarction.

The Effect of a Painful Stimulus and its Recall upon Respiration in Psychoneurotic Patients. By JACOB E. Finesinger (by invitation) and Stanley CoBb, Boston, Mass.

Respiratory records were made by means of a Benedict-Roth metabolism apparatus upon a series of twentytwo psychoneurotic patients of various types and upon a control series of nine subjects, all of whom were under basal conditions. After a preliminary period of three minutes, the pain stimulus was applied for a period of from two to three minutes. The record was continued and five minutes after the end of the stimulation period the patient was asked to recall the ideas and feelings present during the pain stimulus. The suggestion to recall the experience was repeated every thirty seconds for a period of three minutes. The pain stimulus in some experiments consisted of the intracutaneous injection of one half to two cc. of saline in the arm and in other experiments the pain stimulus was repeated pricking of the skin of the forearm with a twenty gauge intravenous needle. The rate of respiration, the depth of the inspiration and expiration, the minute respiratory volume, the oxygen consumption and the type of breathing were noted. Comparisons were made between the preliminary and stimulation periods, the preliminary and recall periods, and between the stimulation and recall periods.

A comparison between the preliminary period and the stimulation period showed that during the stimulation period there was an increase in rate, a decrease in depth of respiration, an increase in minute respiratory volume and an increase in oxygen consumption in over two-thirds of the patients. The same held true for a majority of the control subjects. However, the mean increase in rate, minute respiratory volume, and oxygen consumption was more than twice as great in the patients as in the control subjects.

A comparison between the preliminary and recall periods showed that during the recall period there was an increase in rate, a decrease in depth of respiration and an increase in minute respiratory volume in most of the patients as well as in the control subjects. One half of the patients showed an increased oxygen consumption during the recall period while the other half showed a decrease. All of the control subjects showed a decrease in oxygen consumption. Here too the change observed in the patients was considerably greater than that of the control group.

A comparison between the stimulation period and the recall period showed a decrease in rate, a decrease in depth, and a decrease in oxygen consumption during the recall period in both patients and control subjects. Seven of the patients showed an average increase in minute respiratory volume of 3.0 liters per minute (36 per cent). That is, they were ventilating more when thinking of the stimulus experience than during the actual period of stimulation. Fifteen of the patients showed a decrease during the recall period, averaging 1.7 liters per minute (17 per cent). Of the control subjects two showed an increase, average 0.08 liter per minute ( 2 per cent), while eight showed a decrease of 0.8 liter per minute (13 per cent). There was a marked tendency toward the formation of acute angles at the end of the expiration phase during the stimulation period and during the recall period in both patients and control subjects.

These experiments indicate that psychoneurotic patients reacted much more intensely to the pain stimulus and to the recall of the painful experience than did control subjects.

The Concentration of Serum Uric Acid in Non-affected Members of Gouty Families. By ЈонN H. TALBOTT and (by invitation) FreDERICK S. COOMBS, Boston, Mass.

This communication is a summary of certain data obtained from the study of the immediate families of 16 patients suffering from gout. The clinical diagnosis of gout in the affected members was based upon a history of recurrent attacks of acute arthritis, the finding by $\mathbf{x}$-ray of areas of diminished density in the phalanges of the hands or feet and a concentration of uric acid in the serum greater than $6.0 \mathrm{mgm}$. per cent in two or more samples. In 11 of the 16 patients crystals were recovered from subcutaneous tophi which were morphologically similar to those of sodium urate.

Sixty-eight non-affected members of the families of these patients were investigated and certain studies performed. Only those observations on blood relatives, i.e., parents, siblings, children, or grandchildren are included. All of the non-affected members were thought to be healthy and in none was a past history of acute arthritis obtained. X-rays were taken of the feet of all subjects, and in some, of the hands and knees as well. The concentration of uric acid in the serum was less than 6.0 mgm. per cent in 54 of this group. In the other 14, the uric acid concentration was greater than $6.0 \mathrm{mgm}$. per cent. The concentration of nonprotein nitrogen was below $35 \mathrm{mgm}$. per cent in each sample of blood and it was not believed that the elevated uric acid was a manifestation of renal insufficiency. Several of the non-affected members with an elevated uric acid were less than 30 years of age and it is possible that the metabolic dysfunction began at or shortly after birth. These data lend support to the hypothesis that gout is a familial disease and that one manifestation of it, i.e., an elevation of serum uric acid, may be subject to hereditary transmission.

Carbohydrate Metabolism in Chronic Infectious Hepatitis. By Jerome W. CoNN (by invitation) and L. H. Newburgh, Ann Arbor, Mich.

The nature of the disturbed carbohydrate metabolism in chronic infectious hepatitis was investigated. Six pa- 
tients were studied. All exhibited periods of spontaneous hypoglycemia. Calorimetry demonstrated normal oxidation of glucose under all of a variety of conditions. Yet, the glycemic response to ingested glucose was grossly abnormal. In one phase of the disturbance the response simulated that observed in diabetics; in another phase that found in hyperinsulinism. Carbohydrate restriction always caused severe hypoglycemia. Other indications of disturbed liver function were present. Biopsy or autopsy material from these cases showed different stages of the same pathologic picture, namely, chronic cholecystitis and ascending infectious hepatitis leading to early biliary cirrhosis. In no instance was abnormality of the pancreas demonstrated.

The nature of this disturbance in the metabolism of carbohydrate is usually missed. Impaired hepatic glycogenesis and glycogenolysis appear to account for the abnormality. Such cases have been wrongly interpreted as diabetes mellitus, hyperinsulinism, and dysinsulinism.

Removal of the source of the hepatitis improves the metabolism of carbohydrate. Hence, patients whose symptoms are found to be the result of spontaneous hypoglycemia and who exhibit low fasting blood sugar levels and postprandial hyperglycemia, should be investigated for the presence of biliary tract infection.

\section{READ BY TITLE}

The Etiological Relationship Between Water Retention and Arterial Hypertension in Pregnancy. By MAURICE B. Strauss, Boston, Mass.

The term "toxemia of pregnancy" has been used to designate instances of essential hypertension, pyelonephritis, glomerulonephritis, and malignant nephrosclerosis occurring concomitantly with pregnancy. There is, however, a syndrome in pregnancy consisting of arterial hypertension, albuminuria, edema, headache, vertigo, and visual disturbances, not due to any of the above conditions. Untreated, this syndrome not infrequently terminates in eclampsia. A study of 60 cases of this syndrome has shown that all the signs and symptoms may be increased by any measures which increase water retention and may be relieved by any measures which completely rid the patient of undue water retention.

Water retention in pregnancy has been studied. Anemia, heart disease, and glomerulonephritis may play a rôle in certain cases. With these exceptions, however, it has been shown that the amount of water which will be retained by a pregnant woman under the influence of sodium administration is inversely proportional to the osmotic pressure of the plasma proteins, and the amount of water which will be lost under the influence of a sodiumfree régime is also inversely proportional to the osmotic pressure of the plasma proteins.

The conclusion is drawn that the syndrome of true "pre-eclampsia," including the phenomenon of arterial hypertension, is due to water retention, and that this is in turn chiefly dependent on the osmotic pressure of the plasma proteins.
A Mechanism of Secretion of the Intraocular and Cerebrospinal Fluids. By Jonas S. FrIEDENwaLd and (by invitation) Robert D. STIEHLER and Louis FlexNer, Baltimore, Md.

Observations indicate that in the ciliary body and choroid plexus an electric current causes cations to move from stroma to epithelium and anions to move in the opposite direction. The electron conductor is an oxidation-reduction system in the basement membrane between stroma and epithelium. The energy for this current is supplied by oxidative and reductive processes. The evidence for these views is :-1. At physiological $\mathrm{pH}$ selected basic dyes pass rapidly from stroma to epithelium, but not in the reverse direction, selected acid dyes pass rapidly from epithelium to stroma but not in the reverse direction. Under asphyxia basic dyes pass in both directions with difficulty, acid dyes in both directions with ease. 2. The basement membrane can be studied under asphyxia. Basic dyes pass this membrane with difficulty on the alkaline side of its isoelectric point, and easily on the acid side. The opposite is true for acid dyes. The isoelectric point of the membrane can be shifted reversibly by oxidation and reduction. 3. Indophenol oxidase is present in high concentration in the epithelium but is absent in the stroma. The aerobic oxidation-reduction potential of the epithelium is 0.1 volt more positive than that of the stroma.

Blood Lipoids in Patients with Pituitary Disease Contrasted with Those of Patients with Disorders of the Brain Stem. By EDwIN F. GILDEA, and (by invitation) Evelyn B. Man and John P. Peters, New Haven, Conn.

The blood serum lipoids in 22 patients with many kinds of dysfunction of the pituitary gave no indication that this gland is important in the control of cholesterol, phosphatides, or fatty acids. Seven of 8 patients with various degrees of acromegaly had lipoids that were within or slightly above the normal range. The same was true for 2 patients with pituitary basophilism. Normal for the methods ranges from 150 to $260 \mathrm{mgm}$. per cent for cholesterol, 7.5 to $11.5 \mathrm{mgm}$. per cent for lipoid phosphorus, 9 to $15 \mathrm{~m}$. eq. for titrated fatty acids. Eleven patients with pituitary tumors (adenomas, cysts, ependymoma) had lipoids within the normal range in 9 instances, and moderately high in 2 .

In the second group of 19 patients presenting a complex variety of symptoms many of which could be attributed to disorders of the hypothalamus or other nuclei in the brain stem (extreme vasomotor instability, tachycardia, muscle tremors, mood disturbances) 12 had extraordinarily high lipoids, the cholesterol ranging from 312 mgm. per cent to $615 \mathrm{mgm}$. per cent, the phosphatides from 13.1 to $21 \mathrm{mgm}$. per cent, and the fatty acids from 13.6 to 60 m.eq. Six of the 12 patients had persistent glycosuria that could not be entirely controlled by insulin. Removal of the thyroid only slightly reduced the metabolic rate and gave little symptomatic relief. The remaining 7 had normal lipoids but 2 who were studied repeatedly showed extraordinary fluctuations. 
These results indicate that the pituitary does not play an important rôle in control of blood lipoids. On the other hand, patients with brain stem disorders frequently have extreme degrees of hyperlipemia and extraordinary fluctuations in lipemia.

\section{Redistribution of Blood in Heart Failure. By ARTHUR M. FishberG, New York, N. Y.}

Coexistence of collapsed veins in the extremities with engorged jugular veins was observed in extreme failure of the right heart. The phenomenon was investigated by direct measurement of the simultaneous antecubital and external jugular venous pressures.

Antecubital and external jugular pressures are usually equal in right-sided failure. But with aggravation, usually in the last days, antecubital may fall far below jugular pressure. While jugular pressure remains high, antecubital pressure may fall unmeasurably low. In extreme instances jugular pressure exceeds $20 \mathrm{~cm}$. of water with simultaneous antecubital under $1 \mathrm{~cm}$. With improvement (which is exceptional), antecubital rises to jugular pressure.

The phenomenon is interpreted as a manifestation of vasoconstriction in the extremities evoked (reflexly?) by extreme diminution in cardiac output, i.e., as an analogue in heart failure of what was long ago demonstrated in experimental shock. The selective vasoconstriction results in redistribution of blood, a smaller portion of the cardiac output being distributed to the limbs and a larger to the brain and presumably other viscera.

Symmetrical peripheral gangrene was observed twice. It is suggested that the rare symmetrical gangrene of heart failure (e.g., mitral stenosis with ball-valve thrombus) is angiospastic in origin.

The observations show that venous pressure in the limbs does not necessarily parallel central venous pressure, and that abnormally low venous pressure in the limbs is not always due to primarily peripheral failure.

The Significance of Electrocardiographic Changes Associated with Attacks of Angina Pectoris. By JoSEPH E. F. Riseman, Morton G. Brown, and John V. WALLER (introduced by Herrman L. Blumgart), Boston, Mass.

Continuous electrocardiographic tracings taken during exercise in twenty patients with angina pectoris showed that the characteristic S-T changes associated with attacks of pain become evident long before pain develops and that the disappearance of these changes bears no constant relationship to the disappearance of pain.

Electrocardiographic changes during attacks induced by breathing approximately 10 per cent oxygen while at rest were identical with those observed in the same patients during attacks induced by exercise.

The amount of work which patients could perform before developing an attack of angina pectoris could be increased appreciably by having them breathe pure oxygen continuously both before and during exertion. The characteristic S-T changes induced by a given small amount of exercise could be prevented by having the patient breathe oxygen before and during exertion.

It is apparent, therefore, that the electrocardiographic changes observed during paroxysms of angina pectoris are due to myocardial anoxemia.

Capillary Permeability as Measured by the Pressure Plethysmograph: Observations on Normal Controls, Pathological Controls, and Patients with Severe Intrahepatic Disease. By Benjamin V. White, Jr. (by invitation) and Chester M. Jones, Boston, Mass.

The pressure plethysmograph of Landis was used to measure changes in the volume of a segment of the forearm. A series of preliminary measurements of "reduced arm volume" under a pressure of $200 \mathrm{~mm}$. $\mathrm{Hg}$ was made. The venous return from the arm was then obstructed for ten minutes, after which followed a final determination of the "reduced arm volume." The venous outflow was impeded with a pneumatic cuff, the pressure being greater by a constant amount than the colloid osmotic pressure of the serum as determined by a formula developed by Wies and Peters. The ratio of the increase in arm volume to the actual arm volume as determined by displacement was expressed as a decimal: i.e., the increase in arm volume per cc. of arm volume in ten minutes. The temperature of the plethysmograph was maintained at $35^{\circ} \mathrm{C}$.

A wide range of normal readings was not exceeded in the pathological states studied. Women were found to show rates which approached the higher limits of normal; men, the lower limits. Patients with intrahepatic disease showed rates falling within the limits of normal for their sexes. Patients with hyperthyroidism showed a fall in rate after thyroidectomy, while those with myxedema showed an increase after thyroid administration.

These data affirm the conception that hydrostatic and colloid osmotic pressure changes alone are adequate explanation for edema formation in patients with intrahepatic disease. A pathological change in permeability of the capillaries is apparently not a factor.

Abnormal T-Waves of the Electrocardiogram in Young People Apparently Free of Heart Disease; Their Relation to Posture, Exercise, Infection, and Cardiac Function. By PaUL D. WhITE and (by invitation) Francis L. Chamberlain, Boston, Mass.

Twenty-five patients, apparently free of heart disease, had electrocardiographic abnormalities of the type usually interpreted as indicative of an abnormal myocardial state. Late inversion or low amplitude of the $\mathrm{T}$-waves in Leads II and III, and occasionally in Lead I, were the most commonly noted abnormalities. The electrocardiogram usually became normal with changes of position or after exercise. Electrocardiographic abnormalities reversible with changes in posture were often seen as transient phenomena associated with respiratory infections. The patients were mostly young adults of a nervous temperament and of asthenic habitus. Myocardial function, electrolyte balance, and heart position in relation to various body positions were studied and 
their relation to the electrocardiographic changes is discussed.

\section{Effect of Aminophyllin on the Coronary Capillary Blood} Flow. 1 By Jay Conger Davis, Minneapolis, Minn.

In order to determine in what manner, if at all, aminophyllin might influence coronary blood flow, experiments were carried out using the heart-lung preparation of the dog. Minute flow determinations through the circumflex coronary artery were made with a Stromuhr. In order to determine what factors were involved in case any change occurred, the hot wire anemometer was used so that one could analyze changes, if any, taking place in a single cardiac cycle. Since the canulated coronary artery and its branches on the surface of the heart is kept at practically uniform distention with elimination of pulsation by a blood supply from a perfusion bottle at a constant pressure, any increase or decrease in flow recorded will be due to variations in flow through the coronary capillaries.

The following data were obtained in representative experiments.

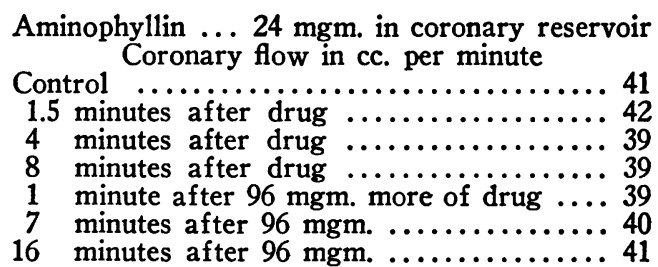

It can be seen that with both small and large doses of aminophyllin no significant changes in coronary blood flow occurred as measured with a Stromuhr. Moreover the hot wire records failed to demonstrate any significant change. Definite evidence of changes in coronary capillary blood flow was found by the use of known constrictors such is pitressin, and known dilators such as sodium nitrite, and drugs that change the strength of cardiac contraction thereby reducing flow in systole such as adrenalin and digitalis.

\section{The Effect of Acetylcholine on the Mammalian Heart as} Studied by Means of a New Piezoelectric Manometer. By A. G. Macleod, New York, N. Y.

A new piezoelectric manometer has been devised which makes possible the recording of intracardiac pressures in intact animals. Recordings are obtained from the right auricle and right ventricle by passing a cannula down the jugular vein, and from the left ventricle by way of the carotid artery. If the cannula is insulated save for its tip, electrograms from the inside of the heart can be recorded simultaneously with the pressure curves.

By means of such simultaneous records the effect of acetylcholine was studied. There is a marked inotropic effect on the auricle but a very slight one on the ventricle. The effect on the electrograms is not marked but con-

1 This work was supported by a grant from the Research Council of the American Medical Association. sists primarily of a shortening. The effects of the various arrhythmias which develop have been observed. Continuous records showing the onset, course, and cessation of auricular fibrillation have been obtained.

An Experimental Study of Cardiac Pain with Reference to the Tension Factor. By S. J. Martin (introduced by L. W. Gorham), Albany, N. Y.

The efficacy of the mechanical factor on coronary arteries in the initiation of cardic pain has been studied in a series of dogs recovering from anesthesia and 4 to 6 hours after surgical preparation according to the Sutton and Lueth procedure. The technique was modified, however, in that 5 black silk ligatures were used in each animal: 3 passing through the adventitia of the anterior descendens branch of the left coronary artery, about $90^{\circ}$ apart, and in the same plane, a fourth and more distal ligature underneath this vessel, and a fifth ligature through the myocardium and visceral pericardium. All ligatures were led to the exterior of the thoracic cage through separate flanged glass tubes sewed in place in the parietal pericardium with the proximal ends of the tubes extending freely into the pericardial cavity. Tension upon the ligatures was applied for different periods of time, but was released as soon as the pain response was noted. The criteria accepted as signifying the presence of pain in dogs awakening from anesthesia were essentially similar to those reported by Sutton and Lueth.

It was found that a characteristic pain response can be elicited when tri-directional tension on the ligatures in one plane (ligatures $1,2,3$ ) is applied to a coronary vessel so as to cause no significant change in blood flow. With this procedure the chemical factors caused by impaired blood flow are completely eliminated, as shown by the absence of changes in the electrocardiogram. The minimal threshold of tension on coronary ligatures was 3 grams in the series of dogs used. Tension on the ligature surrounding the coronary vessel (ligature 4) produced pain and electrocardiographic changes within one minute; tension on the myocardial ligature (ligature 5) produced only a sinus tachycardia and no pain. Local application of alcohol was found to block stimuli initiating cardiac pain, whether they be mechanical or chemical in origin. It is concluded that tension on the coronary arteries in dogs may serve as an adequate stimulus in the initiation of cardiac pain.

A Comparative Study of the Effects of Prolonged Bed
Rest in the Treatment of Congestive Heart Failure. By David Davis, Boston, Mass.

Although the value of absolute bed rest in the course of congestive failure is undisputed, there is a difference of opinion as to how long rest should be continued after evidences of failure have disappeared. In practice, patients are frequently permitted out of bed within a few days after signs of failure have disappeared, and only rarely is absolute bed rest continued for as long as six weeks.

To determine the effects of more prolonged rest, the 
results in a group of patients treated in this manner were compared with those in a group of unselected hospital cases who had recovered from a first attack of congestive failure. The hospital patients were generally permitted out of bed within one to three weeks after symptoms of failure had disappeared. The group receiving more prolonged rest were kept entirely in bed for three to twelve months depending upon the severity of the illness, and periodic prophylactic bed rest was prescribed. Of eleven patients treated in this manner, seven have thus far maintained uninterrupted good health for periods of seven, six, five, three, three, one, and one years respectively. Four have died. The deaths in two of these, who had bundle branch block of long standing, was sudden and unassociated with congestive heart failure.

Of the group of fifty hospital patients, forty had subsequent readmissions for congestive failure. Twentynine of these had a second attack of failure within one year, twenty within six months, and fourteen within three months after discharge. The course of the remaining ten cases with but one admission was followed subsequently in six cases, and these showed a similar course. The longest interval from the time of the first to the second attack of heart failure in the hospital group was three and one-half years.

Seven of the group of eleven patients treated with prolonged rest maintained improvement for three or more years. Only two of the fifty hospital patients showed freedom from congestive failure for as long as three years.

These results indicate that the present-day program of rest for patients with congestive failure is inadequate.

Further Observations Concerning Cardiac Anaphylaxis. By E. Cowles Andrus and (by invitation) Herbert B. Wilcox, JR., Baltimore, Md.

In a previous communication 1 the manifestations of anaphylaxis in the isolated heart of the guinea pig were described and defined. Further studies have yielded the following additional information.

(1) Repeated doses of antigen (horse serum) engender a condition in which the animal survives the intravascular administration of large doses thereof, but the heart of such an animal, even though removed immediat $\in$ ly after such an injection, reacts to small amounts of antigen with typical anaphylaxis.

(2) The hearts of animals sensitized to two antigenshorse serum and egg albumen-are demonstrably sensitive to both and may be desensitized to one and retain the capacity to react to the other.

(3) The probable rôle of histamine or histamine-like substances in cardiac anaphylaxis is supported by certain species differences. In the guinea pig and rabbit both anaphylaxis and the administration of histamine result in constriction of the coronary arteries. In the cat both bring about increased coronary flow.

1 Wilcox, H. B., Jr., and Andrus, E. C., J. Exper. Med., 1938, 67, 169.
(4) The hearts of guinea pigs passively sensitized to Pneumococcus Type I react with typical anaphylactic phenomena following exposure to the pneumococcus polysaccharide Type I (Felton) in quantities as small as $1 / 10,000 \mathrm{mgm}$.

(5) Cardiac anaphylaxis is characterized by two types of reaction which are partially independent.

(a) Alterations in rate and rhythm, and

(b) Changes in coronary flow which parallel the effects of histamine in different species.

The authors desire to record their thanks to Dr. Lloyd D. Felton for the pneumococcus polysaccharide furnished by him.

The Electrocardiographic Signs of an Infarct Located in the Lateral Wall of the Left Ventricle. By F. C. WoOD, C. C. WOLFERTH and (by invitation) S. BeLLET, Philadelphia, $\mathrm{Pa}$.

Pathological studies have shown that, in general, there are three main sites of cardiac infarction: (1) The an. terior-apical, (2) the posterior-basal, and (3) the "mid ventricular," located in the left lateral wall of the heart.

The electrocardiographic signs of the first two have been established. The evidence here presented suggests that the third, less common, type may also be recognizable electrocardiographically.

This series consists of eighteen cases, three of which came to necropsy. The electrocardiographic characteristics are: The RS-T segment is displaced upward in Lead IV (old technique) and downward in Leads I and II. Q III is not seen unless present before the attack. When the lesion heals, all $\mathrm{T}$-waves may return to normal. Thus a healed infarct in this region may be electrocardiographically silent.

There is a high incidence of auricular fibrillation in this series (eight of eighteen cases). Digitalis therefore often confuses the diagnosis of this type of infarct.

The recognition of the fact that a lateral infarct may cause this type of electrocardiographic pattern, has removed one source of considerable diagnostic uncertainty. On the basis of electrocardiographic study it is now possible to classify most large cardiac infarcts as anterior, posterior, or lateral.

\section{The Effect of Carotid Sinus Stimulation Upon Intra}

Cardiac Conduction. By G. LouIs Weller, Jr. (introduced by Henry A. Christian), Washington, D. C.

The present paper describes the results obtained following manual stimulation of the right carotid sinus in man. With a continuous electrocardiogram, taken during the period of stimulation, the cases fall into three groups. In the first group, are those cases in which the numbers of impulses originating from the S-A node are decreased. In the second group although occasionally there is slight effect upon the S-A node, there is a gradually increasing block between the $\mathrm{S}-\mathrm{A}$ and $\mathrm{A}-\mathrm{V}$ nodes. The maximum 
value of prolongation of the $\mathrm{S}-\mathrm{A} A-\mathrm{V}$ conduction time before onset of complete block is remarkably constant for any given case and also seems constant from case to case. This prolongation is 0.30 second maximum, following which complete heart block begins. In the third group, the degree of S-A A-V block is increased until, following complete block, an idioventricular rhythm begins. The sites of origin of the idioventricular rhythm undergo the same sequence of shifts with each new sinus stimulation.

An interpretation of the significance of these changes is not attempted at present. It is believed, however, that one cannot at present draw conclusions as to the functional status of the conduction system and the myocardium from the test, as has been reported in cases of diphtheria.

$A$ Direct Method for Determining Venous Pressure; Relationship of Tissue Pressure to Venous Pressure. By George E. Burch and William A. Sodeman (introduced by R. H. Turner), New Orleans, La.

Indirect and direct venous pressures were determined at heart level for the same portion of the same vein on the dorsum of the hand of 10 normal subjects. The subcutaneous tissue pressure was measured in the same area. The correlation of such determinations has never been reported. The indirect venous pressure measurements were made according to the method of Hooker. The tissue pressure was measured by the technic we have previously described. The direct venous pressure determinations were made with this same apparatus, using a 2 per cent solution of sodium citrate. This method offers the following advantages:(1) the small needle makes it possible to study directly the venous pressure in small veins; (2) negative pressure may be determined; (3) it is simple and accurate; and (4) the lateral openings in the needle make it possible to measure true lateral pressure, eliminating the Bernoulli phenomenon.

Invariably, the indirect determinations were lower than the corresponding direct determinations. The mean indirect value was $8.4 \mathrm{~cm}$. of water, and the mean direct value $11.2 \mathrm{~cm}$. of water. The mean tissue pressure determination was $2.9 \mathrm{~cm}$. of water. After taking the venous pressure with the needle in situ we milked the vein of its blood without stretching the tissues. The intravascular pressure did not drop to zero but closely approximated the tissue pressure. Furthermore, when the values obtained by the indirect technic were added to the tissue pressure values, results closely approximated the direct venous pressure determinations.

The data indicate that: (1) The application of the tissue pressure apparatus to the direct determination of venous pressure was highly satisfactory and disclosed definite advantages. (2) Comparison of indirect and direct venous pressure determinations on the same vessel shows that the former are in error by approximately the tissue pressure. This factor becomes increasingly important as venous pressure decreases.
Observations Concerning the Rôle of the Venous Pressure, Heart Rate, and Total Blood Volume in the Dynamics of the Circulation in Obstructing Pericarditis. By C. Sidney Burwell and (by invitation) Richard H. Lyons, Boston, Mass.

The essential change in the circulation of patients with obstructing pericarditis is an interference with diastolic filling imposed by the pericardial thickening and constriction.

A young man with pericardial obstruction was studied for two years. Following relief of the obstruction by operation comparative observations were made.

In the presence of obstruction: the blood volume was increased to 21 per cent above normal, the venous pressure persistently elevated, the circulation time prolonged, and the stroke volume limited. Spontaneous fluctuations in the blood volume were accompanied by parallel changes in the venous pressure, but not by demonstrable changes in the stroke volume or heart rate. Induced changes in the blood volume (phlebotomy, transfusion, or infusion) were accompanied by marked changes in the venous pressure but no change in the stroke volume, heart rate, circulation time, or arterial pressure. Induced acceleration of the pulse rate (atropine intravenously) was accompanied by a striking and immediate fall in the venous pressure but no change in the stroke volume, circulation time or arterial pressure. Exercise was accompanied by a rise in venous pressure and heart rate, a decrease in circulation time, and no change in the arterial saturation. Hyperventilation was accompanied by no change in the venous pressure in contradistinction to the fall in venous pressure observed in normal people.

Shortly after the relief of the obstruction the venous pressure and the blood volume were within normal limits and the cardiac output higher than before.

The Cardiovascular Effects of Intravenous Injections of Fifty Per Cent Dextrose and Sucrose in Normal Persons and in Patients with Heart Failure. By LAURence B. Ellis and James M. Faulkner, Boston, Mass.

The effects on the circulation of the intravenous injection of $100 \mathrm{cc}$. of 50 per cent dextrose or sucrose were studied in a large group of subjects. These included subjects with normal cardiovascular systems and patients with various types of heart disease in all stages of heart failure. The cardiovascular effects of the injection of dextrose and sucrose were similar. There was no significant change in the arterial blood pressure or in the heart rate. The venous pressure tended to rise during the course of the injection, the average increase being three centimeters of water and the maximum seven centimeters. In most instances it began to fall promptly when the injection was stopped and had reached the control level in 20 to 30 minutes. An increase in the blood plasma volume also took place promptly. The increase within one minute of the end of the injection, which took ten minutes, averaged 12 per cent with a maximum of 20 per cent. The dilution of the blood plasma lasted longer than the increase in venous pressure, and tended to be 
maintained for about 30 minutes but had usually disappeared at the end of one hour. This increase in plasma volume, in excess of the amount of fluid injected, was due to the drawing of fluid from the tissue spaces into the blood stream by the hypertonic action of the sugar introduced. No significant differences in the response of the circulation could be detected depending on the degree of heart failure. No untoward symptoms occurred in any of the patients studied with the exception of a few mild chills. The clinical implications of this study are discussed, particularly as relating to the strain thrown on the circulation by such injections, especially in patients with impaired cardiovascular systems, and regarding the efficacy of dextrose and sucrose in increasing the blood volume.

The Nature of Hypertension in Coarctation of the Aorta.

By J. Murray Steele and Alfred E. Cohn, New York, N. Y.

In this demonstration it is shown by use of Hamilton's direct method of recording intra-arterial pressures that the normal levels in the femoral artery are no higher than those in the radial. In a case of coarctation of the aorta which exhibits systolic and diastolic hypertension in the arms, and many symptoms of essential hypertension, such as headache, nervousness, and emotional instability, measurements of pressures in the femoral and radial arteries have been made by direct puncture, and simultaneous records obtained. Contrary to what is believed to be the case it is now found that although the pulse pressure is exceedingly low in the femoral artery (11 to $15 \mathrm{~mm} . \mathrm{Hg}$ ) the diastolic level (108 to 120 ) is almost as high as in the arms (120 to 130$)$. The systolic pressures were 120 to 130 in the femoral, and 180 to 192 in the radial arteries.

The observation suggests that peripheral resistance may be as greatly increased in the legs as in the arms in these individuals and casts doubt upon the belief that increased vascular resistance occurs only in the upper half of the body. The phenomenon of hypertension in coarctation would then appear to be closely analogous to that obtained by Goldblatt by clamping the aorta above the point of emergence of the renal arteries.

\section{Experiences with Thiocyanate Therapy in Vascular $H y$ -} pertension. By Edward Massie, Clayton B. EthRIDGE, and ROGER W. ROBINSON (by invitation) and JAmes P. O'HARE, Boston, Mass.

During the last three years sodium and potassium thiocyanate have been administered to 45 cases of uncomplicated vascular hypertension. Dosage was regulated by the blood cyanate level according to the method of Barker. Fifteen cases were first observed under carefully controlled conditions for nine months. During the first three months, the patient received no therapy of any kind; in the second, he received the drug in a simple vehicle; during the third period the drug was removed from the vehicle without the patient's knowledge. With the blood cyanate concentration maintained between 5 and
$7 \mathrm{mgm}$. per cent, results were good with relief of cerebral symptoms in 12 of 15 cases and with an average fall in pressure ranging from 66 to $21 \mathrm{~mm}$. systolic and 33 to $8 \mathrm{~mm}$. diastolic. During the second control period symptoms recurred and pressures rose to former levels. Serious toxic effects were noted in two patients, consisting of weakness, nausea, and vomiting in one and an initial attack of angina pectoris in another.

Our aim in the second group of 30 less closely controlled patients was to extend our experiences with the drug and to see if a greater fall in pressure would result with larger doses. With the blood cyanate level ranging between 8 and $15 \mathrm{mgm}$. per cent, a greater fall in pressure was noted with an average of 78 to $10 \mathrm{~mm}$. systolic and 40 to 0 diastolic. Three cases showed no appreciable drop. Five cases had a maximum fall of over $100 \mathrm{~mm}$. systolic. There was symptomatic relief of headaches in 17 out of 19 cases. Coincident with the higher dosage, more toxic effects were observed. Nine cases showed marked weakness, 2 had hallucinations, 2 developed angina pectoris, 1 had congestive heart failure and 1 had a severe dermatitis.

We believe that thiocyanate therapy when carefully controlled is a valuable therapeutic agent in hypertension.

\section{Supradiaphragmatic Splanchnic Resection for Essential} Hypertension. By Robert Sterling Palmer and (by invitation) Reginald H. Smithwick, Boston, Mass.

Observation of sixty-five patients with essential hypertension, who have been submitted to supradiaphragmatic splanchnic resection during the past two years leads one to conclude that there is a forty to fifty per cent chance of a good result in patients under forty years of age who have no organic changes, or at the most mild to very moderate changes and who exhibit very labile blood pressures under observation. There is a very poor chance of obtaining a good result in patients older than this with any considerable degree of organic change. The progress of hypertensive heart disease is not prevented. The onset or progress of renal failure is not prevented and, from the indication of one patient, the onset of the malignant phase of essential hypertension is not prevented. One patient with possible old pyelonephritis was much helped, while another was not helped at all. Two cases with the menopausal type of hypertension have apparently received great benefit.

While at this time we are making no attempt to evaluate the procedure on the basis of symptomatic relief, it is worth commenting that in almost every case with severe headaches a very marked and striking benefit has been obtained, regardless of whether or not a favorable effect on the blood pressure was obtained. We have no explanation for this. Symptomatic relief otherwise is not more than is commonly obtained, even in the severe cases by appropriate medical measures. Theoretical considerations and animal experimentation indicate that this procedure is unlikely to be of benefit in essential hyper- 
tension. What interests us is that practical observation shows that within the limits of observation, a very favorable effect is obtained in 40 to 50 per cent of early and, in some cases, of moderate essential hypertension. Whether or not some of these cases have an extreme degree of vasomotor instability rather than true early essential hypertension, we cannot definitely say. It is our opinion that all the cases operated on have had true essential hypertension.

The Kidney Function in Hyperthyroidism. By ElMER C. Bartels (by invitation) and Frank N. Allan, Boston, Mass.

A study of the urea clearance test was made in a series of twenty-three cases of hyperthyroidism excluding cases in which there was evidence of hypertension, vascular degeneration, or any suspicion of primary renal damage. In six cases there was some degree of impairment of kidney function, but this appeared to be related to age, since all but one of the patients were over 50 years. The urea clearance was not influenced by the severity or duration of hyperthyroidism in younger individuals.

Reaction of Renal Blood Flow to Partial Constriction of the Renal Artery. By HENRy A. Schroeder (by invitation) and Alfred E. CoHn, New York, N. Y.

Arterial pressure and renal blood flow were measured in dogs while the renal artery was partially constricted. Systolic and diastolic pressures were recorded by Hamilton's manometer, renal blood flow by a thermo-stromuhr modified from that of Schmidt. The clamp and the thermo-stromuhr were aseptically placed on a dog's renal artery. Further observations were made one or two days later while the renal artery was being subjected to various degrees of clamping.

It was found that when the renal artery was partially clamped, renal blood flow immediately fell but after a few minutes rose again slowly almost to its previous level without corresponding changes in blood pressure. Further clamping was followed by a rise in flow which was, however, smaller than the first. Four increases in the degree of constriction have been followed by successively diminishing rises in blood flow. When the clamp was released, this phenomenon was observed to occur again on subsequent clampings. A small rise in diastolic pressure, however, was sometimes noticed when the artery was constricted to a point at which blood flow did not return toward normal. If it is assumed that the flow of blood is proportional to the logarithm of movement of the recording galvanometer, then it would appear that the blood flow must be reduced to roughly one-third before the subsequent rise in flow fails to take place. Some intrarenal vascular reflex is considered responsible for this phenomenon.

This result extends to an internal renal adjustment a mechanism which Rein noticed on modifying the systemic blood pressures.
The Recovery of an Adrenalin-like Substance from the Kidney. By T. R. HARRISON and (by invitation) J. R. Winliams, Jr., and E. B. Grossman, Nashville, Tenn. Kidneys of hogs and dogs were perfused with Ringer's solution. Such perfusate was found to have a pronounced pressor effect when injected into rats. The rise in blood pressure set in quickly and was of short duration. It was heat stable and dialyzable. Its action was enhanced by cocaine and diminished by ergotamine. The pressor effect obtained was of the same order as that produced by $1: 1,000,000$ to $1: 10,000,000$ adrenalin solution. The renal perfusate also caused dilatation of the pupil of the enucleated eye of the frog. It is believed that the pressor principle recovered from the kidney is either adrenalin or some substance having properties closely resembling those of adrenalin. Since it was found that the perfusate obtained immediately after removal of the kidney contained less pressor principle than perfusates made several hours after the removal of the kidney it is thought that the blood pressure raising substance may be a degradation product of renal tissue. When a similar perfusion technique was applied to the other organs no pressor principle was recovered.

\section{A Study of the Sodium, Potassium, Chlorine, and Water Exchange in Relation to Nephritic Edema. By R. H.} Freyberg, F. R. White and F. D. Lathrop (introduced by P. S. Barker), Ann Arbor, Mich.

The effects of the ingestion of sodium chloride, sodium bicarbonate, potassium chloride, potassium bicarbonate, and ammonium chloride on the exchange of sodium, potassium, chlorine, and water were studied in a patient with chronic hemorrhagic nephritis with edema, and compared with responses of two control subjects under identical conditions.

During the administration of sodium chloride and later sodium bicarbonate, nearly all of the sodium was retained by the nephritic and was slowly eliminated when the administration of these salts ceased. This response contrasted sharply with the behavior of the control subjects in whom only small amounts of sodium were retained during the administration of sodium chloride and sodium bicarbonate, and all that was retained was quickly eliminated after the administration of these salts was stopped. In all three subjects water was retained in amounts corresponding to the retention of sodium. When potassium chloride and potassium bicarbonate were administered, practically no potassium was retained by the nephritic or control subjects, and no large or consistent change in the water exchange was observed. The administration of ammonium chloride was attended by an increased elimination of sodium, potassium, and water in the nephritic as well as in the controls.

The Rate of Removal of Hemoglobin from the Circulation and its Renal Threshold in Human Beings. By Reuben Ottenberg and (by invitation) Charles $L$. Fox, JR., New York, N. Y.

The primary object of the work was to establish with some precision normal standards for human beings. 
Human hemoglobin was prepared entirely free from stromata. Twenty normal persons received intravenous injections of varying amounts ( 3.47 to 8.25 grams) without regard to blood grouping. There were no untoward symptoms.

A method was devised for the collection of blood plasma with absolutely no manipulative hemolysis. No bilirubinemia was developed. This is explicable by the small amount of bilirubin produced and the perfect liver function.

The initial concentrations of hemoglobin in the plasma were dependent directly on the plasma volume. The rates of removal of hemoglobin from the plasma after large and small doses of hemoglobin were different. After large doses it was very rapid during the first three hours, suggesting some special mechanism. The amount of urinary hemoglobin was insufficient to explain its rapid removal from the plasma during the first hours. As compared with other substances the rate of removal of hemoglobin from plasma is slow: it required ten or more hours for the plasma to be cleared.

Hemoglobinuria occurred in eleven cases. In terms of plasma concentration the renal threshold for human hemoglobin was found to be very variable. Hemoglobinuria usually occurred when the plasma concentration exceeded $200 \mathrm{mgm}$. per $100 \mathrm{cc}$. But in three cases it occurred at concentrations as low as 142, 146, and 177 $\mathrm{mgm}$., and in six cases failed to occur in concentrations over $200 \mathrm{mgm}$. per $100 \mathrm{cc}$. of plasma.

In terms of dose of hemoglobin per kilogram of body weight a more constant renal threshold for hemoglobin was found. Unexpectedly it was different for the two sexes. In women it was $72 \mathrm{mgm}$., in men $92 \mathrm{mgm}$. per kilo.

The experiments throw some light on the mechanism of hemoglobinuria. The theory of Lichty, Havill, and Whipple is tentatively accepted: according to this there is a "glomerular threshold" i.e. a plasma concentration above which hemoglobin is filtered through the glomeruli; it is rapidly absorbed by the renal tubular epithelium and and only when the rate of filtration exceeds the $a b-$ sorbtive capacity of the tubules is hemoglobin found in the urine.

Serum Sulfate Concentrations in Renal Insufficiency and Patients on Inadequate Fluid Intake. By E. G. WAKEfield, Marschelle $H$. Power (by invitation) and N. M. KeIrH, Rochester, Minn.

Determinations of the concentration of blood urea, serum sulfate, and values for urea clearance in a series of patients with renal insufficiency showed substantial agreement. A comparison of these same tests in cases in which the urine had a specific gravity of 1.025 or more for a twelve-hour specimen disclosed interesting variations which were probably dehydration phenomena. Serum sulfate concentrations in cases in which patients had taken insufficient amounts of water are summarized.
The $p H$ of the Urine as Influenced by the Ingestion of Alkaline Mineral Waters. By Walter S. McClemLAN and (by invitation) ROBERT GoLDSTEIN, Saratoga Springs, N. Y.

The authors have determined the $\mathrm{pH}$, by Taylor's colorimetric standards, of 500 specimens of urine, following the ingestion of plain water and of mineral waters which contain from 2.0 to 5.0 grams of the bicarbonates of sodium, calcium, and magnesium per liter. The subjects carried on their work and took their usual meals.

For control, the specimens taken hourly through the day either without water or after plain water, showed a $\mathrm{pH}$ of 5.0 to 6.0 in the early forenoon, ranged from 6.5 to 7.2 between 10 a.m. and 1 p.m., and in the afternoon varied from 5.0 to 6.5 .

After taking 500 or $1,000 \mathrm{cc}$. of mineral water following breakfast, the urinary $\mathrm{pH}$ by 11:00 to 12:00 o'clock reached 7.4 to 8.2 and in the afternoon ranged from 6.0 to 7.5. When the mineral water was taken in divided amounts through the day, the urinary $\mathrm{pH}$ varied between 6.5 and 8.0 throughout the 24 hours.

The greater the diuresis, the more nearly the $\mathrm{pH}$ approached 7.0. The shift into the alkaline range was directly proportional to the amounts of bicarbonates in the different mineral waters studied.

The Effect of Alcohol and of Alcoholic Liquors on the Normal and Pathological Kidney. By Maurice Bruger, Nober W. Guthrie, and S. Arthur Localio (introduced by Carl H. Greene), New York, N. Y.

The urinary protein, urine volume, Addis count, urea clearance, and urea ratio were studied before and after one to three doses of 12 to $50 \mathrm{cc}$. of pure ethyl alcohol given in 25 per cent solution or as whiskey. Sixteen patients with nephritis and nephrosclerosis and five normals were studied.

Results. 1. An occasional increase in the Addis count was observed in the five normals after the ingestion of alcohol; this was not elevated above the normal range. No proteinuria or impairment of renal function was observed. 2. Alcohol did not augment the proteinuria in patients with nephritis or nephrosclerosis. 3. Moderate diuresis was induced even in patients with marked impairment of renal function. 4 Alcohol did not increase the excretion of red cells and casts in seven patients with chronic nephritis and in three of four patients with acute nephritis. The ingestion of alcohol or whiskey by five patients with nephrosclerosis produced a transient increase in the Addis count. 5. Renal function remained unchanged after the ingestion of alcohol in acute and chronic nephritis, and did not delay recovery in two patients with acute nephritis manifesting a gradual improvement in renal efficiency. In four of five patients with nephrosclerosis, the impairment in renal function was increased temporarily by the ingestion of alcohol. 
Studies of the Principle in Liver Effective in Pernicious Anemia. $V$. Additional Accessory Factors and Further Properties of the Primary Factor. By Y. SuBBAROW (by invitation) and BERNARD M. JACOBSON, Boston, Mass., and (by invitation) Stanley J. HARTFALL, Leeds, England.

We have recently presented evidence for the view that the therapeutic action of liver extract in pernicious anemia depends upon the presence of a primary factor (or factors) exerting by itself only slight hematopoietic effect, and upon the presence of at least three chemically distinct accessory factors which, in the absence of the primary factor, are therapeutically inert but which markedly augment the activity of the primary factor. These accessory factors include $l$-tyrosine, a complex purine, and a peptide.

Over one year ago we described certain chemical properties of the primary factor, derived from a Fuller's earth filtrate of commercial liver extract and obtained in a yield of $2 \mathrm{mgm}$. from 100 grams of liver. By further purification the total organic solid content of the yield of the primary factor was reduced to $1.2 \mathrm{mgm}$. from 100 grams of liver. This material, together with the three accessory factors, has been administered parenterally to eight patients, in dosages varying from 0.14 to $0.64 \mathrm{mgm}$. per day. The resulting hematopoietic responses were moderate in three patients, slight in two patients, and were completely negative in three patients. Certain evidence suggested that the diminished potency of this preparation of the primary factor was not due to the administration of inadequate amounts of the factor, but rather was conditioned by the absence of additional accessory factors. Two such materials were isolated from the mother fraction of the primary factor, and were identified as tryptophane and guanosine, respectively. These two substances together with the three above-mentioned accessory factors, but without the primary factor, were administered to one patient in a dosage of each substance derived from 50 grams of liver per day. This mixture proved completely inert. On the other hand, the administration to eight patients of these five substances together with the primary factor, in dosages varying from 0.12 to $0.26 \mathrm{mgm}$. per day, resulted in considerably greater potency than that effected by the above-described mixture of primary factor with the three accessory factors, but devoid of guanosine and of tryptophane. The hematopoietic responses were good in six patients, slight in one patient, and negative in the remaining patient. In four of these patients the administration in second periods of large amounts of commercial or of crude experimental liver extract did not result in further reticulocyte responses. Finally, this primary factor was further purified by readsorption on charcoal, elution, and precipitation by a mixture of alcohol and ether, with resulting considerable diminution in its content of organic solids. This material, in an amorphous form, was administered to one patient in a dosage of $0.06 \mathrm{mgm}$. per day, together with the five accessory factors, each derived from 20 grams of liver per day. The resulting response was good.
The chemical properties of the primary factor suggest that it is a complex pyridine derivative. Further evidence for this view is furnished by the therapeutic effect of a simple pyridine derivative represented by nicotinic acid amide. To one patient was administered parenterally synthetic nicotinic acid amide in a dosage of $2 \mathrm{mgm}$. per day, together with three accessory factors (tyrosine, complex purine, and peptide). To a second patient was administered $1 \mathrm{mgm}$. of nicotinic acid amide per day, together with the same accessory factors. In both cases the hematopoietic responses were moderate, and were accompanied by clinical improvement.

Response to Liver Extract of Experimentally Induced Anemia in Rabbits: Negative Results. By AdoLPH J. Creskoff (by invitation) and Thomas Fitz-Hugh, JR., Philadelphia, $\mathrm{Pa}$.

The production of severe macrocytic anemia accompanied by leukopenia and thrombopenia in rabbits injected with deproteinized typhoid bouillon concentrate was recently reported by Wolf, Weber and Kröger. ${ }^{1}$ According to these authors, if the animals are treated beforehand or concurrently with daily parenteral liver extract, anemia still appears but despite continued toxin administration a reticulocyte crisis occurs between the eighth and twelfth days, with subsequent recovery of erythrocyte, hemoglobin, leukocyte, and thrombocyte values. The reticulocyte response is described as being entirely similar to the classical crisis seen in pernicious anemia in humans. These experiments have been repeated by us.

Typhoid bouillon concentrate was prepared and injected daily in various dosages, together with liver extract, into a series of rabbits. In no case did macrocytic anemia, leukopenia, or thrombopenia appear. The reticulocyte responses were slight and irregular in onset and duration. A control rabbit showed practically comparable reticulocyte fluctuations.

Other investigators have described a pernicious anemialike blood picture produced with B. Welchi toxin.2, 8 These experiments were performed prior to the advent of liver therapy. The effect on the blood of injections of perfringens toxin was, therefore, investigated with a view also of testing liver extract in the treatment of these animals.

Rabbits given a single intravenous dose of perfringens toxin were found to develop severe anemia of hemolytic type within a few hours. This anemia was promptly succeeded by a regenerative blood picture showing a high reticulocyte percentage. These immature cells appear to be responsible for the "macrocytosis" reported by the above investigators. Subcutaneous injection of the toxin induced a lesser and more slowly developing anemia.

1 Wolf, H. J., Weber, C., and Kröger, E. Med. Welt, 1937, 11, 1170.

2 Kahn, M. C., and Torrey, J. C. Proc. Soc. Exper. Biol. and Med., 1925, 23, 8.

${ }^{8}$ Reed, G. B., Orr, J. H., and Spencer, C. M. J. Infect. Dis., 1927, 41, 282. 
Liver extract given before or concomitantly with the toxin did not protect against its effect nor was the rate of blood regeneration influenced.

Conclusion. 1. Toxins prepared from typhoid and perfringens bacillus do not engender true macrocytic anemia when injected into rabbits. 2. The anemia thus produced does not respond specifically to liver extract.

The Blood Picture in Rats with Experimental Liver Damage. By R. W. Heinle, Cleveland, Ohio (by invitation), and W. B. CAstLE, Boston, Mass.

Cirrhosis of the liver with splenomegaly was produced in rats by the administration of carbon tetrachloride vapor. As in similar experiments by Higgins and Stasney, a severe macrocytic anemia resulted. Insofar, however, as the blood picture was characterized by reticulocytosis, leukocytosis, and spontaneous recovery, it was quite unlike pernicious anemia although, as in pernicious, and experimentally produced severe hemolytic anemias, the bone marrow was hyperplastic and in some instances megaloblastic in type. The anemia is interpreted as being of hemolytic origin, the macrocytosis probably resembling that seen clinically in certain other acute hemolytic anemias. Parenteral liver extract administered at the height of the anemia produced only slight or no significant increase in an already high reticulocyte level. Aminopyrin given in various doses in the presence of severe liver damage did not produce leukopenia or other manifestations of agranulocytosis.

Studies in Bone Marrow Decompensation: the Hypoplastic States. By Charles A. Doan and (by invitation) Carr V. Moore and Ben C. Houghton, Columbus, Ohio.

Bone marrow is an organ. As such its hemopoietic functions are affected by many diverse factors, any one or any combination of which may lead at times to varying degrees of decompensation. The resulting anemic states reflect a relative marrow hypoplasia as varied in etiology as those factors which may contribute to myocardial decompensation. Marrow recompensation may occur spontaneously or at times may be therapeutically induced if the contributing factors in the individual case can be discovered and influenced favorably.

With careful analyses the proportion of so-called primary or idiopathic aplastic states may be reduced to a minimum. When encountered they are characterized by a progressive marrow panhypoplasia, by a high transport plasma iron (see previous iron studies), by a normal plasma for growth inhibiting or growth stimulating substances as measured by blood cell cultures in vitro, and by a refractoriness to all known hematopoietic stimuli. Splenectomy (physiologic inhibitor-see previous studies) performed in selected cases, especially in those presenting early thrombocytopenic purpuric manifestations, has resulted in cellular recompensation for varying periods up to two years. In other instances repeated massive blood transfusions from polycythemic donors have coincided with prolonged remissions.
In those hypoplastic states secondary to some determinable or discoverable toxic inhibiting or destructive agent, as benzol, a more or less complete recovery may follow if the influence can be eliminated before too extensive mesenchymal damage has been effected. The bone marrow picture is very different in these cases from that seen in the "idiopathic" aplastic states. In those individuals showing a persistent (11/2 years), though no longer progressive, deficiency of circulating marrow elements, the removal of a normal sized, physiologically active spleen we believe to be a rational procedure, and in our experience, such therapy has been followed by a normal cellular reequilibration persisting indefinitely.

In adolescence, an endocrine imbalance may be associated with the hypoplastic syndrome and an important part of the treatment consists in a correction of these deficiencies. In some hypoplastic states there is found a moderate degree of generalized lymphocytic hyperplasia in nodes and bone marrow reciprocal to the myeloid and erythroid hypoplasia, which directs attention to another possible mechanism (see previous studies).

\section{Yellow Bone Marrow in the Treatment of Primary} Granulocytopenia. By Charles H. Watkins (by invitation) and Herbert Z. Giffin, Rochester, Minn.

During experimental studies with the administration of bone marrow for secondary anemia in 1928 and 1929, a moderate increase in the number of monocytes and neutrophils was observed in blood smears. This observation led to the administration of bone marrow in cases of granulocytopenia. The first case of agranulocytic angina was treated with bone marrow in July 1930. Since then twenty-four patients with agranulocytic angina or primary granulocytopenia have been treated, fourteen of whom are now living. In three cases in which subsequent death occurred no bone marrow was given during the fatal attack at home, although it had been administered with apparent success during an earlier attack. Five patients who died received an inadequate dose of the material; the dose was inadequate because the degree of ulceration and edema of the tissues of the throat prevented the ingestion of sufficient quantities of bone marrow. Only two patients who received an adequate dose of bone marrow died, and in these cases very severe complications were present. In this group of twenty-four patients bone marrow was given during twenty-nine attacks in all. In only twenty-one attacks was an adequate dose administered; the number of attacks with adequate dose and recovery was nineteen, and the number of attacks with adequate dose and death was two.

During the acute stage from 200 to $\mathbf{4 0 0}$ grains of bone marrow were given daily; one patient took as much as 800 grains daily. As monocytes and polymorphonuclears increased in the blood smears the dose was decreased. Bone marrow was administered with apparent success in some of the early cases without withdrawal of drugs now known to be related to the etiology of agranulocytic angina; in certain other cases there was no history of the ingestion of such drugs. Therefore the results cannot be 
entirely attributed to the withdrawal of drugs to which the patient may have been sensitive.

The Inefficacy of Congo Red in Hemolytic Anemia. By GarnetT F. Cheney (by invitation) and W. Dock, San Francisco, Cal.

The administration of Congo red, intravenously, to two cases of congenital hemolytic icterus, was followed in only one by a striking rise and fall in the reticulocyte count. In two cases of acquired hemolytic anemia there was no significant change in the reticulocyte count, and in none of the four cases was there any rise in red cell count or in hemoglobin. While the drug is of no value in treatment of such cases, it is remarkable that in cirrhosis, congenital hemolytic jaundice, and pernicious anemia it may cause a marked reticulocytosis in a small proportion of cases. The fall in serum bilirubin noted in two of the cases of pernicious anemia makes it appear unlikely that reticulocytosis was due merely to "irritation" of the myeloid tissue.

\section{Atypical Hereditary Hemorrhagic Syndromes. By AR-} thur J. Geiger, New Haven, Conn.

A man with severe hemophilioid bleeding exhibited prolonged bleeding time with increased capillary fragility, but with normal platelet counts and normal clotting phenomena. Study of the immediate family revealed five similarly afflicted individuals of both sexes in three successive generations. Six reports of similar syndromes in the world literature were critically reviewed, and a composite delineation of the disorder presented. The prognosis is poor, the mortality from hemorrhage is appreciable, and the patients are bad surgical risks. Transfusion during the bleeding episodes constitutes the most reliable therapeutic measure. The heredity pattern is of the simple dominant type. Pathogenesis apparently involves primarily a functional platelet abnormality and a supplementary vascular defect attributable to a degenerated inheritable anlage.

An additional critical review of other atypical hereditary hemorrhagic syndromes suggests that all these disorders are intermediate forms of hemorrhagic disease whose extremes are represented by hemophilia on the one hand and idiopathic thrombocytopenic purpura on the other. Data is available in support of this concept. The general designation "hereditary hemophilioid purpura" is suggested for the entire group of atypical hereditary hemorrhagic disorders, and a system of terminology applicable to the various individual syndromes is proposed.

Effects of Refrigeration on Adult Human Blood Mixed with Various Preservatives. By ELMER L. DEGowin and Everetr D. Plass (introduced by Horace $M$. Korns), Iowa City, Iowa.

The current interest in the use of preserved blood for transfusion makes imperative studies of the changes which blood undergoes during refrigeration over extensive periods of time and with the use of various preservatives. Adult human blood was mixed with various preservatives and maintained at temperatures of 2 to $4^{\circ} \mathrm{C}$. for 20 to 30 days. Samples were studied at approximately 5-day intervals and the following determinations made: Amount of free hemoglobin in the plasma; potassium content of the plasma; corpuscular resistance to hypotonic solutions; corpuscular resistance to mechanical manipulation; changes in corpuscular volume; changes in glycolytic properties of the corpuscles. With these data it was possible to evaluate the relative merits of various preservatives and to determine the maximum time of preservation during which blood could safely be used for transfusion.

The Hypophysis and Hematopoiesis. By OvID O. MEYER and (by invitation) Ethel W. Thewlis and Harold F. Rusch, Madison, Wis.

Previously it has been demonstrated that hypophysectomy in rats was succeeded by anemia and a pronounced diminution in reticulocytes. Furthermore, in these experimental animals, polycythemia, reticulocytosis, and bone marrow hyperplasia did not result from the stimulus of oxygen want as in control rats. Reticulocytosis without attendant increases in the hemoglobin or erythrocytes followed the administration of antuitrin growth hormone, however.

Further studies are now to be reported in an attempt to prove or disprove the original premise that the hypophysis has some control, possibly hormonal, over hematopoiesis. It is now established that though neither antuitrin growth hormone nor oxygen want produce an increase in all three components, i.e., hemoglobin, erythrocytes, and reticulocytes, the administration of both resulted in a response resembling that of normal rats to oxygen want. The administration of antuitrin growth hormone which has been previously boiled for thirty minutes, thereby destroying the growth factor, resulted in loss of most, though not all, of the reticulocyte stimulating factor. Thyroidectomy was followed by a decrease in reticulocytes, but the change was not nearly so striking as in hypophysectomized rats. The administration of antuitrin growth hormone to thyroidectomized rats was not attended by more than a slight reticulocytosis, but the stimulus of oxygen want resulted in a response analogous to that which occurred in normal rats. The administration of thyroxin to hypophysectomized rats resulted in a significant, though somewhat delayed increase in reticulocytes. However, as with the antuitrin growth hormone, there was no increase in hemoglobin or erythrocytes, but rather a persistent or progressive anemia.

The administration of thyrotropic hormones to hypophysectomized rats produced reticulocytosis similar in degree to that of thyroxin.

These observations suggest that neither the hypophysis nor the thyroid alone is responsible for the described hematological changes. The results depend upon removal of the hypophysis but possibly by withdrawal of a thyrotropic factor. The growth hormone of the hypophysis is not solely responsible for the hematological changes. 
Observations on the Interrelationship of Macrocytic Anemia, Hypoproteinemia, and Hepatic Disease. By Frank H. Bethell, Jean Kyer, and Gerald Rottschafer (introduced by Cyrus C. Sturgis), Ann Arbor, Mich.

Quantitative blood studies and serum protein determinations were made on 26 pregnant women and 148 cases of chronic debilitating disease of various types, controlled by similar studies on a series of healthy men and women. A high incidence of macrocytic anemia was found in the presence of lowered protein values, particularly in patients with hepatic damage. In an attempt to elucidate the interrelationship of these changes rats were maintained on protein deficient diets. Progressive lipoidosis of the liver cells occurred, which, during the added strain of pregnancy, became extreme. The pregnant rats with very severe liver damage developed macrocytic anemia, a megaloblastic reaction of the bone marrow, and low serum protein values. Deficiency of the vitamin B complex apparently played no part in these results, nor could they be prevented by antipernicious anemia liver extract. It is concluded that macrocytic anemia and hypoproteinemia are coincident effects of impaired liver function and that hepatic damage may be caused by dietary deficiency.

The Relationship Between the Erythrocyte Sedimentation Rate and the Plasma Proteins. By Marian W. Ropes and Elsie Rossmeisl (by invitation) and Walter Bauer, Boston, Mass.

The clinical value of the erythrocyte sedimentation rate has been established, but the factors underlying variations in rates remain obscure. The majority of investigators have concluded that fibrin is the chief factor. However, the occasional occurrence of marked inconsistencies between the concentration of fibrin and the rate has led some workers to conclude that there is not a causal relationship.

The present investigation includes studies of the sudden, marked changes in rate occurring in acute gonorrheal arthritis and the more gradual changes observed in rheumatoid arthritis. In general, blood samples with rapid rates showed high concentrations of fibrin and globulin. There was a tendency toward a linear relation between fibrin content and rate, but there were many marked exceptions. Blood from one case of rheumatoid arthritis during an acute exacerbation showed a 300 per cent rise in rate, while fibrin and globulin values remained at the upper limit of normal. In one case of multiple myeloma a 67 per cent increase in fibrin and a 12 per cent increase in globulin was associated with a 27 per cent drop in rate.

These and other studies indicate that variations in rates cannot be ascribed to any one known factor. The explanation with which all of the findings are consistent is that the rate is dependent on the colloidal state of the serum, which, in turn, is affected by the concentration of individual colloids.

We conclude that there is not a quantitative correla- tion between the sedimentation rate and the concentration of fibrin, globulin, or total protein.

\section{Changes in the Volume of the Blood and Plasma during} Exercise. By Nolan L. Kaltreider (introduced by Wm. S. McCann), Rochester, N. Y.

The blood volume and its components, the hematocrit, serum proteins, hemoglobin, red blood cells, viscosity, and oxygen consumption were determined in normal subjects and patients with heart disease before, during, and after performing a given task on a stationary bicycle ergometer. Normal subjects performed on an average $404 \mathrm{kgm}$. per minute for 10 minutes; the patients with heart disease, 85 per cent of this work.

During exercise the volume of the blood and plasma of normal subjects decreased on an average of $255 \mathrm{cc}$. (4.6 per cent) and $245 \mathrm{cc}$. ( 8.0 per cent) respectively; the cell volume, $20 \mathrm{cc}$, while the hemoglobin, the hematocrit, and serum proteins increased on an average of 0.9 gram, 2.0 per cent, and 0.64 gram per 100 cc. of blood respectively. Twenty-five minutes after exercise the blood and plasma volumes were slightly larger than during the control period. The diminution in the plasma volume during exercise is roughly proportional to the increase in the venous pressure. In one subject as the work was increased there was a further decrease in the plasma volume.

In the patients with heart disease, at rest, the values for the plasma and blood volumes were larger than the predicted ones. During exercise the average blood and plasma volumes decreased $185 \mathrm{cc}$. (3.3 per cent) and 235 cc. (7.9 per cent) respectively, while the total cell volume increased $25 \mathrm{cc}$. The hemoglobin, hematocrit, and proteins increased 0.9 gram, 2.6 per cent, and 0.53 gram per $100 \mathrm{cc}$. of blood respectively. In contrast to normal individuals, the blood and plasma volumes of the patients with heart disease were still moderately decreased, twenty-five minutes after exercise.

These results show that in normal subjects during exercise there is hemoconcentration, with a transfer of fluid from the vascular system to the tissue spaces. It appears that this transfer of fluid is due to an increase in the hydrostatic pressure of the blood. In normal individuals during exercise there is no evidence that new cells are added to the circulation, while in heart disease there is a slight increase in the total cell volume.

The Toxicity for Human Beings of Diethylene Glycol with Sulphanilamide. By TheOdore G. KLUMPP and (by invitation) Herbert O. CALvery, Washington, D. C.

In September and October 1937 approximately 203 gallons of a proprietary remedy "Elixir of Sulphanilamide" were distributed. It was composed essentially of 75 per cent diethylene glycol and 8 per cent sulphanilamide. Data on 104 deaths associated with the consumption of this drug are available for toxicological study. It may be summarized as follows : 


\begin{tabular}{|c|c|c|c|c|c|c|c|c|}
\hline Age group & 兽 & 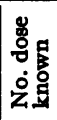 & 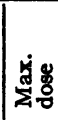 & 邑总 & 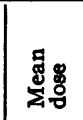 & ชే & 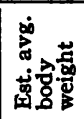 & $\overline{0}$ \\
\hline $\begin{array}{l}7 / 12-11 \text { yrs.. } \\
17-25 \text { yrs... } \\
25-\ldots \ldots \ldots\end{array}$ & $\begin{array}{l}33 \\
23 \\
48\end{array}$ & $\begin{array}{l}33 \\
20 \\
43\end{array}$ & $\begin{array}{r}c c . \\
120 \\
150 \\
240\end{array}$ & $\begin{array}{l}c c . \\
13 \\
30 \\
20\end{array}$ & $\begin{array}{r}c c . \\
51.8 \\
88.7 \\
102.2\end{array}$ & $\begin{array}{l}32.4 \\
30.4 \\
42.2\end{array}$ & $\begin{array}{l}70 \\
80\end{array}$ & $\begin{array}{l}1.3 \\
1.3\end{array}$ \\
\hline
\end{tabular}

Information on the human toxicity of sulphanilamide and experimental pharmacological and toxicological studies in animals suggest that the toxic effects of the socalled elixir of sulphanilamide were essentially due to the diethylene glycol contained in the preparation.

Observations on the Chemotherapeutic Effect of Sulfanilamide in Experimental Hemolytic Streptococcal Infections. By M. H. DAwson, and (by invitation) G. L. HobBy, New York, N. Y.

There has been much speculation concerning the mechanism of the action of sulfanilamide and related compounds in hemolytic streptococcal infections. Some investigators have stated that these drugs are more effective against virulent strains than against avirulent. Others have expressed the opinion that the effect of the drug is primarily directed against the toxins and hemolysins produced by the organisms.

In studying the problem we have arrived at the following conclusions :

(1) Sulfanilamide is equally effective against virulent and avirulent strains.

(2) Sulfanilamide does not affect either the production or the activity of soluble hemolysins.

(3) The erythrogenic toxin is not neutralized by the drug.

(4) Sulfanilamide is effective against hemolytic streptococci of Groups A, B, C, E, F, and G. It exerts little or no effect on Group D organisms.

(5) The drug is effective against non-hemolytic strains within Group B.

(6) The drug is effective anaerobically as well as aerobically.

(7) The drug owes its activity to bacteriostatic rather than bactericidal properties. In this respect it does not differ from other bacteriostatic agents. Its unique character is due to the fact that it is not neutralized in the presence of tissue or body fluids.

\section{Neutralization of Vaccine Virus by Specific Immune} Serum. By Robert F. PARker, Cleveland, Ohio.

Addition of serum from an animal immune to vaccinia to a suspension of active vaccine virus neutralizes the ability of the virus to cause infection when the mixture is inoculated intradermally in a rabbit. The present studies indicate that for a given serum concentration the amount of virus neutralized depends upon the amount of virus added to the mixture but that it is not a fixed proportion of this, for as the amount of virus added is increased, proportionally more of it is neutralized. Similarly the amount of virus neutralized by various concentrations of serum is regular, and the results of titrations are reproducible within reasonable limits. With increasing dilution, the relative effectiveness of serum in neutralizing virus appears to increase regularly. The curve obtained by plotting the amount of virus neutralized against the concentration of serum is a rectangular hyperbola. The data at hand do not provide an adequate basis for explanation of the mechanism of neutralization of vaccine virus by immune serum, but they do indicate definitely the regularity of this action.

Studies on Serum Proteins. II. Identification and Determination of Three Immunologically Distinct Globulins in Human Sera. By Forrest E. KeNDALL (introduced by Arthur J. Patek, Jr.), New York, N. Y.

Normal human serum has been shown to contain three different globulins, which have been called alpha-, beta-, and gamma-globulin. Preparations of alpha- and gammaglobulin have been obtained essentially free from the other components. Antisera which react specifically with each of the three globulins have been prepared and have been used to determine the amounts of each of the globulins present in the serum of normal individuals and of a limited series of patients with cirrhosis of the liver, chronic glomerular nephritis with edema, and rheumatoid arthritis. The average values for the amount of these globulins in normal individuals are: Alpha-globulin 1.4 grams per $100 \mathrm{ml}$; beta-globulin 0.7 gram per 100 ml.; gamma-globulin 0.35 gram per $100 \mathrm{ml}$. Not only are the amounts and proportions of these globulins changed in the diseases studied but evidence is also found that additional varieties of globulin are present in many cases.

An Experimental Investigation of the Penetration of Antiseptics through Tissues. ${ }^{1}$ By ARTHUR D. HIRSCHFELDER and (by invitation) MILAN Novar, Minneapolis, Minn.

Since penetration of antiseptics through tissues had never been investigated, we tested the penetrating power of various antiseptics by injecting $0.05 \mathrm{cc}$. of bouillon culture of pooled staphylococci into the tissues, applying the antiseptic to the surface, and subsequently dissecting out the area containing the cocci, grinding, diluting, plating, counting and comparing with the symmetrically situated control area, over which no antiseptic had been applied.

Punctured wounds were made in rabbit's thighs with nails of various diameters, the cocci introduced into the puncture $6 \mathrm{~mm}$. below the surface and the antiseptic applied to the surface. None of the antiseptics listed below, when applied to the surface even as a wet dressing continuously for 24 hours, reduced the number of living cocci unless the diameter of the puncture was more than $4 \mathrm{~mm}$. and the wound was actually gaping. The walls of the puncture close together and act as a perfect valve to prevent penetration.

\footnotetext{
1 Supported by a fund from the Eli Lilly Company.
} 
When the cocci were injected subcutaneously under the guinea pig's skin and the antiseptic solution was applied to the skin above them (separated from the cocci by a thickness of about $1 \mathrm{~mm}$. of tissue) none of the antiseptics applied to intact skin, burned skin, or skin injured by clamping with a haemostat penetrated sufficiently to kill the subcutaneous cocci. Electrophoresis increased penetration only when the current was strong enough to coagulate the tissues.

There was some penetration through scarified guinea pig's skin, but when the antiseptic solution was only painted upon the scarified skin none of the antiseptics killed as many as $\mathbf{5 0}$ per cent of the staphylococci injected subcutaneously. However, application of wet dressings of the following solutions continuously for 4 hours killed 90 per cent or more of the staphylococci injected $1 \mathrm{~mm}$. below the surface of scarified guinea pigs' skin: Phenol 3 per cent; iodine 5 per cent; $\mathrm{HgCl}_{2}$ 1:2000; neutral acriflavine $1: 1000$; gentian violet $1: 1000$; alcoholic merthiolate $1: 1000$; alcoholic mercurochrome 1:100; alcoholic metaphen 1:1000. One per cent $\mathrm{AgNo}_{3}$ and also Dakin's solution did not penetrate at all. Granulation tissue 48 hours old completely blocked penetration.

All these antiseptics delayed healing, caused leukocytic infiltration, edema, and some necrosis. Intracutaneous injection of $0.1 \mathrm{cc}$. caused sloughing.

We propose as the criterion of antiseptic efficiency the Antiseptic Efficiency Coefficient $=$

Lowest intrademal sloughing concentration

Lowest penetrating antiseptic concentration For phenol this is $\frac{1 \text { per cent }}{3 \text { per cent }}=0.33$, and by taking this coefficient for phenol as the unit, we propose also the Phenol Efficiency Coefficient, the ratio of the Antiseptic Efficiency Coefficient to that of phenol. For all the antiseptics tested this Phenol Efficiency Coefficient was never greater than 1.5 even though their phenol bacteriostatic coefficients in vitro ranged from 0.7 to 500 .

The Development of Bactericidal Properties by the Blood of Normal Rabbits after Repeated Bleedings. By C. Phinlip Minler, Chicago, Ill.

Normal adult rabbits (free from snuffles and coccidiosis) were bled $10 \mathrm{cc}$. from the heart once or twice a day for three or four days. The bactericidal action of the series of whole defibrinated bloods from each rabbit was determined by adding to aliquot portions of each sample progressive dilutions of young cultures of meningococci, and gently agitating them in a rotating machine at $37^{\circ} \mathrm{C}$. for 36 to 72 hours, when they were cultured. In each series the initial sample of blood had the least killing power and subsequent samples showed a significant, progressive increase.

The same increase in bactericidal action was observed in a series of sera from successive bleedings, to which cells were supplied by a previously unbled rabbit. A similar, but smaller increase occurred in series of sera alone.
The bactericidal action was very much reduced by heating the sera to $56^{\circ}$ for thirty minutes.

Conclusion. After repeated bleedings the blood of normal rabbits develops bactericidal properties which depend, chiefly, at least, on a heat-labile component of the serum.

The Diagnosis of Weil's Disease (Infectious Jaundice). Concerning the Use of Guinea Pigs for the Detection of Strains of Leptospira icterohemorrhagiae of Low Virulence. By J. T. SyverTon (by invitation), Grorge Packer Berry, and (by invitation) W. W. Stiles, Rochester, N. Y.

The infrequency with which Weil's disease is being recognized in this country appears to us to be inconsistent with the widespread occurrence in rats of the causative agent, Leptospira icterohemorrhagiae. It seems likely that many cases are escaping detection, and that the explanation may lie in the inadequacy of the "guinea pig method" as usually employed in the laboratory, i.e., the inoculation of guinea pigs with human material and the subsequent recognition of the experimental disease. The ordinary criteria for such recognition have been the development of jaundice, hemorrhages, and death in the inoculated pigs. These considerations led us to begin the present investigation 4 years ago. During this time we have studied 863 guinea pigs infected with 34 strains of Leptospira icterohemorrhagiae.

In a series of 152 guinea pigs inoculated with 2 strains of low virulence, only 1.3 per cent became jaundiced and only 8.5 per cent died. Generalized hemorrhages were found in only 2 per cent and hemorrhages in the lungs in only 31 per cent. It is obvious that the criteria usually accepted for the recognition of experimental Weil's disease in guinea pigs are inadequate. These findings indicate that the majority of guinea pigs inoculated with slightly pathogenic strains of Leptospira icterohemorrhagiae survive. In the diagnostic laboratory such animals are apt to be regarded as negative. The following simple procedure prevents such mistakes. Diagnostically inoculated guinea pigs should be routinely killed on the 12th day after inoculation, and their blood and kidneys used for the inoculation of further guinea pigs. (The test-animals should weigh from 125 to 175 grams. Larger guinea pigs are too resistant, and should not be used.) Three such passages should be carried out before a negative report is made. A few passages suffice to enhance the pathogenicity of organisms of low virulence so that they produce a lethal infection. Of the 152 animals, 84 per cent showed a transient febrile reaction $\left(40^{\circ} \mathrm{C}\right.$. or above). When a febrile reaction occurs in a guinea pig, it is important to bleed the animal by cardiac puncture, and to pass the blood to further guinea pigs. Since this procedure entails some risk, the human material under study should be inoculated initially into several animals.

The simple procedure discussed above has been used successfully for the isolation of a number of strains of Leptospira icterokemorrhagiae from wild rats and from human beings. We would not have recovered the or- 
ganism from some of the cases, if we had not followed the technique described.

Susceptibility of Young Rabbits to Respiratory Infection. By Yale KNeEland, JR., and (by invitation) BARbara Mulliken, New York, N. Y.

In connection with the observed insusceptibility of the newborn human infant to the common cold we noted some years ago that a litter of young rabbits did not develop snuffles until the age of 5 to 6 weeks in spite of the fact that the mother was suffering from a severe case of snuffles during the nursing period. It seemed unsatisfactory to explain this phenomenon on the basis of inherited immunity. Recently we have performed a number of experiments to determine whether the phenomenon occurs regularly in the hope that, if so, further analysis may throw some light on the insusceptibility of the new born to certain infectious diseases.

The extensive studies of Webster established the fact that rabbit snuffles is caused by $P$. lepiseptica. Our bacteriological observations on snuffles in adult rabbits have confirmed the findings of Webster. $P$. lepiseptica is regularly recovered from cases of the disease, and when a pure culture of the mucoid form of this organism was introduced intranasally into 6 previously uninfected adult rabbits, 5 became chronic carriers and of these 3 developed clinical snuffles.

Quite a different effect was observed when similar amounts of the same culture were administered intranasally to 35 infant rabbits between 3 and 4 weeks of age. Clinical snuffles was never produced. From only 7 was the organism ever recovered on nasal culture, and in very small quantity, one or two days immediately following inoculation. The nasal passages of all 35 showed no gross evidence of chronic inflammation at autopsy, and in 34 instances there was no growth on culture. However, 5 of the animals died of pneumonia and suppurative pleurisy from which a pure culture of $P$. lepiseptica was obtained.

These experiments indicate that fully virulent $P$. lepiseptica has no capacity to produce local inflammation of the nasal passages of infant rabbits, and virtually no capacity to survive in this region, although in certain instances it is capable of causing death by general invasion.

\section{Pulmonary Lesions in Dog Pneumococcus Carriers In- fected with Distemper. By Whelan D. Sutriff, New York, N. Y.}

A method was sought for producing pneumococcic pneumonia in dogs without the use of direct intrabronchial inoculation. Intranasal inoculations of as much as $1 \mathrm{cc}$. of virulent Type I pneumococcic broth culture in 2 to 6-months old puppies produced no pneumococcic lesions, although Type I pneumococci were shown to reach the lungs. A carrier state of about 10 days' duration resulted. Distemper was produced in 10 such pneumococcus carriers. When the symptoms of distemper were mild, no pulmonary lesions developed. In 3 animals in whom the clinical manifestations were severe, pneumonia resembling lobar pneumonia appeared in parts of the lungs and a patchy hemhorragic pneumonia appeared in other parts of the same animals lungs. Pneumococci were cultured from homogeneous pneumonic lesions, and hemolytic streptococci, gram negative bacilli and sometimes Type I pneumococci were cultured from patchy hemhorrhagic pneumonic lesions.

The Absorption of Pneumococcus Antibody in NonPneumonic Subjects After Intramuscular Injection of Antipneumococcus Horse and Rabbit Sera. By Maxwell Finland and (by invitation) JoHN W. Brown, Boston, Mass.

Observations were made with Type I and Type V antipneumococcus sera. When an unconcentrated Type I rabbit serum was given intramuscularly, higher titers of homologous antibody-were demonstrated in the circulating blood and these titers were reached sooner than when the same amount of antibody contained in the same volume of concentrated horse serum was injected in the same manner. Both of these serums gave no local nor general reactions. In similar observations with Type $\mathrm{V}$ pneumococcus antibody using concentrated rabbit and horse serum, the rabbit serum gave moderate to severe local reactions and some constitutional symptoms. The titers of circulating Type $\mathrm{V}$ antibody attained by the rabbit serum recipients were lower, especially during the first 24 hours, than in subjects receiving corresponding amounts of antipneumococcus horse serum.

The highest titers of circulating antibody were attained 48 hours after the intramuscular injections. The titers of circulating Type I antibody found 24 to 48 hours after intramuscular injection of rabbit serum were almost as high as those found in other subjects receiving the same amount of antibody, either in horse or rabbit serum, intravenously. Giving the same amount of Type I antibody in horse or rabbit serum, both intravenously and intramuscularly, higher titers persisted over a longer period in those who received the rabbit serum intramuscularly.

Studies on the Action of Sulfanilamide in Vitro. By Chester S. Keefer and (by invitation) Lowell A. Rantz, Boston, Mass.

From previous studies it was ascertained that $\beta$-hemolytic streptococci were killed in whole blood by phagocytosis and intracellular digestion. In order to determine the effect of sulfanilamide on the bactericidal action of whole blood, varying amounts were added to different samples of whole blood and the results compared with those obtained with whole blood alone. The following results were obtained. (1) Sulfanilamide has a marked bacteriostatic effect on $\beta$-hemolytic streptococci, Group $A$, when added to whole blood in amounts of $10 \mathrm{mgm}$. per 100 cc. of blood or more. (2) In many cases in which there was no evidence of bactericidal power in the whole blood alone, there was no increase in bactericidal power of the blood following the addition of sulfanilamide. (3) In some samples of whole blood it was possible to dem- 
onstrate an increase in the bactericidal action of whole blood by adding sulfanilamide. This was usually conspicuous in the samples of blood which contained antibodies.

It would appear from these observations that sulfanilamide has a bacteriostatic effect in whole blood and, in some instances, the addition of the drug increases the bactericidal effect of whole blood. It is suggested that this action is due to a reduction in the rate of growth of the organisms which allows the antibody and cells to act more effectively. Protocols and charts illustrate the effects enumerated.

The Infectiousness of Semen of Patients with Late Syphilis: An Experimental Study. By Jarold E. KEMP (introduced by J. E. Moore), Chicago, Ill.

In an attempt to discover the reasons for the persistence of the belief that semen is more infectious than the other body fluids of individuals with late syphilis, it was found that this opinion originated in the pre-Wassermann era in an effort to explain the apparent immunity to syphilis of the mothers of congenitally syphilitic children. After the discovery of the treponeme this belief persisted because of (1) the experimental observation that experimental animals inoculated elsewhere with syphilis develop metastatic testicular lesions with great frequency and (2) the conclusions of Warthin, who believed that a certain type of testicular interstitial fibrosis was characteristic of syphilis since he had demonstrated treponemes by silver staining in certain such testes.

In an experimental study of this question the semen of fifteen syphilitics whose infection was of four years' duration or more was inoculated intratesticularly into rabbits. These inoculations were all negative. In a review of all the available reports of studies of semen for the presence of treponemes, it was found that, including our fifteen cases, the semen of 144 syphilitics has been investigated by one or more of several experimental methods. Treponemes were demonstrated in 14 (9.7 per cent) of the individuals. In 119 of the 144 it was possible to estimate the duration of the syphilitic infection. In 67 with syphilis of less than four years' duration treponemes were found in the semen of 13 (19.4 per cent). In 52 with syphilis of four years' duration or more treponemes were found in the semen of 1 (1.9 per cent).

The experiments of Kertesz, who has reported a high incidence of the successful inoculation of rabbits by the intravitreous injection of the semen of patients with late syphilis, were repeated and his experimental methods reviewed. His findings could not be confirmed and his methods were found to be at such variance with accepted experimental procedures that his conclusions cannot be accepted.

The results of this study and a review of the literature of related experimental studies indicate that there is at the present time insufficient evidence to support the belief that the semen of individuals with late syphilis is frequently infectious.
The Susceptibility of Freshly Isolated Strains of $S$. pallida to Arsphenamine and Neoarsphenamine in vitro. By Harry EAGLe, Baltimore, Md.

Contrary to general belief, the arsphenamines (arsphenamine, neoarsphenamine, silver arsphenamine, metaminoparahydroxyphenyl arsenoxide) in high dilution immobilize and kill pathogenic $S$. pallida in vitro. Sulpharsphenamine has proved relatively inactive. The original observation was made with the Nichols strain, which has been passed through rabbits for 25 years. Qualitatively similar results have been obtained with six new strains of $S$. pallida freshly isolated from human lesions and tested after only one or two rabbit passages. The effective concentrations of the arsenicals in vitro were of the same order of magnitude as those attained in the body after the therapeutic administration of these drugs. It remains to ascertain whether the actively spirocheticidal agent is the arsphenamine per se or oxidation products formed in vitro under the conditions of the experiment.

Carbohydrate Metabolism in Subjects with Athyrea. By E. C. Cutrer and (by invitation) M. Pijoan, Boston, Mass.

Investigations concerning the effect of hypothyroidism on carbohydrate metabolism have already received considerable study. On the other hand the myxedematous state appearing in its natural form as a result of either glandular or pluriglandular disease and usually described as spontaneous in character varies from the state of myxedema following total thyroidectomy. This is significant when we consider that spontaneous myxedema exists in the presence of the thyroid gland whose disease may be dependent on other glandular disturbances. Thus carbohydrate studies in individuals suffering from spontaneous myxedema may yield conclusions not necessarily similar to those studies made on subjects with total thyroidectomy.

Our subjects were carefully chosen and were divided into two groups, those who had diabetes mellitus previous to the operation and those subjects in whom the operation was performed for intractable heart disease or other vascular disease. At least one year had elapsed since the operation before these studies were carried out and in each instance thyroid extract was withheld from the patients for a period of six weeks. Our investigation consisted in the study of the changes in respiratory quotients, blood sugars and certain associated chemical changes following the oral ingestion of $1 \mathrm{gram}$ per kilo of body weight of dextrose and the intravenous administration of $1 / 2$ unit of insulin per kilo of body weight.

Our results tend to show that following total thyroidectomy there is a marked increased sensitivity to insulin characterized by a speedy fall in the blood sugar with a tendency towards a prolonged hypoglycemic phase. This finding was true in diabetic and non-diabetic subjects with the exception of one patient with scleroderma whose blood sugar was practically unaffected by the intravenous administration of insulin. The sugar time curves were 
in general equivocal but the hyperglycemia following the ingestion of dextrose was usually less than one would expect and was definitely improved in the diabetic individuals as compared to their preoperative sugar time curves.

The Organic-Inorganic Partition of Iodine in Blood of Normal and of Hyperthyroid Individuals. By $\mathrm{H}$. J. Perkin (by invitation) and L. M. Hurxthal, Boston, Mass.

A method for the quantitative analysis of the organic and the inorganic iodine partition of $10 \mathrm{cc}$. of blood has been devised. The accuracy of the procedure has been established by the recovery of known amounts of organic or inorganic iodine added to blood.

Blood iodine fractionation has been carried out in 35 normal individuals, 50 cases of non-toxic adenomatous goiter and 150 cases of primary hyperthyroidism. The quantitative relationship of the organic and the inorganic iodine in the blood of the above groups has been established.

The Problem of Thyroid Tolerance. By W. O. ТномРson and (by invitation) P. K. Thompson, S. G. TAYLOR, III, and L. F. N. DickIE, Chicago, Ill.

The problem of the gradual development of partial tolerance to thyroid derivatives keeps constantly arising and is important in treatment and in biologic assay. It is reported that animals develop a tolerance to thyroxine. In man the data are difficult to obtain and too meager to warrant conclusions. To settle the problem, we have carried out the following two series of observations:

1. Repeated oral administration of the same dose of the same lot of desiccated thyroid to the same patient (5 patients).

2. Repeated intravenous administration of the same dose of racemic thyroxine to the same patient (10 patients).

The total calorigenic response to each dose was observed, the basal metabolism being allowed to return to its original level before giving further treatment.

Our observations do not show the development of a tolerance in man, either to desiccated thyroid administered by. mouth or to thyroxine administered intravenously. Some variation in the response to the same dose of material was noted, but the fluctuations did not follow any trend, the effect being sometimes slightly greater, sometimes slightly less. It follows that, in a thyroidless individual, the same amount of desiccated thyroid or of thyroxine will produce approximately the same response indefinitely.

The Part Played by the Secondary Hyperparathyroidism in the Disordered Calcium and Phosphorus Metabolism in Rickets. By Fuller Albright and (by invitation) Hrrsh W. Sulkowitch, Boston, Mass.

That there is a secondary hyperparathyroidism in most cases of infantile rickets and osteomalacia can be con- sidered as established from evidence obtained by histological study and bio-assay. It was the object of these studies to learn which features of the disordered calcium and phosphorus metabolism are directly a result of the hypovitaminosis $\mathrm{D}$ and which are the result of the secondary hyperparathyroidism.

To unravel this problem it was necessary to find out the effects of each of these factors alone. The direct action of vitamin $D$ was divorced from that due to secondary parathyroid activity by studies on hypoparathyroid patients. These showed that the changes following vitamin $D$ administration are the result of two separate mechanisms: (1) an increased calcium absorption from the gut and (2) an increased urinary phosphorus excretion. Since the action of the parathyroid hormone on calcium and phosphorus metabolism was already pretty well established, it was then possible to construct a chart for rickets showing the part played by the hypovitaminosis and that played by the hyperparathyroidism and the relation of one abnormality to the other. Two of the more important conclusions were that the hypophosphatemia in rickets is a manifestation of the secondary hyperparathyroidism and that the latter tends to be of a degree just sufficient to keep the serum calcium at a normal level.

On the basis of these conclusions it seemed possible to divide cases of hypovitaminosis $D$ into the three following groups: $(A)$ simple hypovitaminosis $\mathrm{D}$ with no compensatory hyperparathyroidism-serum calcium low, serum phosphorus normal; $(B)$ hypovitaminosis $\mathrm{D}$ with a compensatory hyperparathyroidism-serum calcium normal, serum phosphorus low; and $(C)$ hypovitaminosis D with a hyperparathyroidism insufficient to compensateserum calcium low, serum phosphorus low.

Oral Therapy in Adrenal Insufficiency. The Efficacy of a Concentrated Adrenal Cortical Extract Preserved in Glycerol.1 By George W. ThorN and (by invitation) Kendall Emerson, Jr. and Harry Eisenberg, Baltimore, Md.

The disadvantages occasioned by the frequent subcutaneous injections of adrenal cortical extract in patients with advanced Addison's disease are well known. It has been shown that the effect of a single injection of extract is short lived and that a constant supply of hormone can be insured only by repeated injections. ${ }^{2}$ The desirability of administering the extract in some form which provides a more constant rate of absorption is readily understood. It has been observed that glycerol is a good preservative as well as a good solvent for cortin.8 A concentrated adrenal cortical extract pre-

1 Aided by a grant from the Committee on Research in Endocrinology, National Research Council.

2 G. W. Thorn, H. R. Garbutt, F. A. Hitchcock, and Frank A. Hartman, Endocrinology, 1937, 21, 213.

8 Frank A. Hartman, George W. Thorn, and R. R. Durant, Endocrinology, 1937, 21, 516. 
served in glycerol 4 ( 1 cc. of the final glycerol preparation representing 50 grams of original adrenal cortex) has been substituted orally for injected extract in three bilaterally adrenalectomized dogs and in five patients with severe Addison's disease maintained on a constant metabolic regimen. Observations were made regarding the renal excretion and the blood serum concentration of electrolytes, blood nonprotein nitrogen and sugar, body weight, and appetite. Substitution of oral extract was found to result in a maintenance of sodium and chloride balance, body weight, blood pressure and serum electrolyte concentration. Withdrawal of the oral extract was attended by a negative sodium and chloride balance, loss of body weight, reduction in blood pressure, and serum concentration of sodium and chloride. Oral extract therapy prevented the increased renal excretion of sodium and chloride which regularly follows bilateral adrenalectomy in the dog. Oral therapy was effective in maintaining the life of an adrenalectomized dog despite a diet of low sodium and high potassium content. An equal quantity of 50 per cent glycerol solution (control) or of the aqueous extract used for injection was ineffective when administered orally.

The oral extract requirement necessary to maintain adrenalectomized dogs and patients with Addison's disease is two to three times that of the injected extract. It appears that the oral administration of an adequate quantity of adrenal cortical extract suitably prepared is capable of maintaining the life of both adrenalectomized dogs and patients suffering from Addison's disease.

Hormone Assays in a Case of Androgenic Adrenal Cortical Tumor. By Charles L. Hudson (introduced by J. M. Hayman, Jr.), Cleveland, Ohio.

Although reports of cases of virilism syndrome associated with tumors of the adrenal cortex are not extremely rare, a search of the literature fails to reveal any cases where assays of estrin and androgenic substances in the urine of such patients have been done. For this reason a patient recently admitted to the Lakeside Hospital who had the virilism syndrome and from whom an adrenal tumor was removed surgically seems worthy of report.

A 23-year old white female came to the hospital complaining of amenorrhea, acne, hirsutism of the face and body, baldness, and a manly build. A tumor mass above the right kidney was beautifully outlined on $x$-ray after perirenal insufflation of air. The tumor was removed and was stained with, among others, the Ponceau De Zylidine stain, believed by some to be specific for adrenal cortical cells which have the androgenic quality. The tumor gave a positive reaction whereas control adrenal sections did not.

Very high values for estrin and androgenic substances were found before operation. With analyses at weekly intervals after operation, the estrin and androgenic substances were found to have declined to normal levels.

4 Prepared by Doctor David Klein of the Wilson Laboratories, Chicago, Illinois.
Paralleling the change in hormone excretion there have been a resumption of normal menstruation, enlargement of breasts, deposits of fat over the hips, disappearance of acne on the face, change in psyche, and disappearance of some of the excess hair on the abdomen.

Metabolic Changes Produced in a Patient with Pituitary Dwarfism Following the Administration of the Growth Hormone of the Anterior Hypophysis, and After the Addition of Thyroid Therapy. By JAmes A. Greene and (by invitation) J. E. Harris, H. Levine, and R. B. Gibson, Iowa City, Iowa.

Our knowledge of metabolic changes produced in man by parenteral administration of growth hormone of anterior hypophysis is rudimentary. We have studied the calcium, phosphorus, sulphur, and nitrogen balances; blood calcium, phosphorus, and phosphatase ; urinary creatinine and creatine; basal metabolism; and glucose tolerance in a patient with pituitary dwarfism before and at various intervals for one year after administration of the hormone, and after addition of desiccated thyroid gland therapy.

At first, the calcium, phosphorus, and nitrogen retention increased, but the storage of sulphur diminished. The addition of desiccated thyroid therapy produced negative calcium and sulphur balances and reduced storage of nitrogen and phosphorus. Four months later growth hormone was discontinued for one month, and after being resumed, the nitrogen storage was reduced and calcium, phosphorus, and sulphur balances became negative. Addition of desiccated thyroid therapy for seven months produced an increase in patient's height. At this time positive balances for all the minerals and nitrogen were observed, and an increase in the dosage of the growth hormone produced a greater retention of calcium and phosphorus, but a slight reduction in the nitrogen and sulphur. The results of the other studies and the significance of our results will be discussed.

Addison's Disease with Associated Myxedema. By R. A. Cleghorn, Toronto, Canada.

A low basal metabolic rate is frequently found in patients suffering from Addison's disease. Such patients usually show none of the clinical signs of myxedema. Three cases presenting the characteristic signs, symptoms, and blood chemistry of Addison's disease with associated signs and symptoms of true myxedema have been encountered and studied. Thyroid extract in each case raised the basal metabolic rate and relieved the signs and symptoms of myxedema. The clinical findings with results of treatment of these cases are discussed.

The Effect of Dihydrotachysterol in Parathyroid Tetany. by F. W. Sunderman and (by invitation) EDWard Rose, Philadelphia, $\mathrm{Pa}$.

The effects of dihydrotachysterol, a derivative of irradiated ergosterol, have been studied in five patients suffering from postthyroidectomy parathyroid tetany. 
Employing the same diet throughout, the patients were studied during intervals which were divided as follows:

1. A period during which amounts of calcium lactate and viosterol sufficient to control symptoms were administered ;

2. A period during which all medication was withdrawn and during which symptoms of tetany appeared;

3. A period during which dihydrotachysterol alone was administered.

The beneficial therapeutic effects of dihydrotachysterol are reported. During each period of observation studies were made of the non-diffusible and diffusible calcium, inorganic phosphorus, and protein of the serum, as well as the total calcium content of the ingesta and egesta.

The Relation of the Pituitary to Blood Serum Amylase Activity in the Dog. By Oliver Cope, and (by invitation) Anders Hagströmer and Hester Blatt, Boston, Mass.

Six dogs were hypophysectomized by a parapharyngeal approach. On a seventh a control operation was performed, leaving the pituitary undisturbed. The serum amylase activity was measured by a modification of the method of Scharles and Salter. ${ }^{1}$ Extensive observations of the blood serum amylase activity before hypophysectomy confirmed the absence of significant effect of food, fasting, glucose, and insulin. Adrenin was followed by a slight rise in activity. Hypophysectomy resulted in a precipitous rise in activity in the second twenty-four hours, reaching a maximum at forty-eight hours which was maintained throughout life. The longest survival was seventy-two days. This rise did not appear in the operative control. Using the formula of Scharles and Salter the rise represented more than a two-fold increase in activity. The high level of activity was not significantly affected by glucose or spontaneous hypoglycemia. Adrenin caused a further slight increase in one of two animals. The abrupt rise in amylase activity closely parallels the appearance of the increased insulin sensitivity measured in rabbits. It is concluded that the anterior pituitary is a major influence in the control of serum amylase activity.

The Effects of Male Sex Hormone on Menstruation and in the Menopause. By E. Shorr and (by invitation)

G. N. Papanicolaou and B. G. Stimmel, New York, N. Y.

We have previously reported 1 that menstruation can be inhibited in the normal menstruating woman by the male hormone (testosterone propionate). These studies have been amplified by hormonal assays and vaginal biopsies in addition to vaginal smears, and have been extended to the menopausal group.

\footnotetext{
1 Scharles, Frederick H., and Salter, William T., Am. J. Cancer, 1934, 30, 613.

1 Papanicolaou, G. N., Ripley, H. D., and Shorr, E., Proc. Soc. Exper. Biol. and Med., 1938, 37, 689.
}

In the normal menstruating woman, prolonged treatment with male hormone, beginning mid-menstrually, permits the next menses to occur generally earlier than expected, but inhibits the following one. The vaginal smears assume a menopausal character. The vaginal epithelium becomes atrophic. The excretion of gonadotropic substances remains at a low level. The female sex hormone continues to be excreted during treatment at levels which indicate either that ovarian function is not entirely abolished or that there is some transformation of the male into the female sex hormone. The former alternative is favored. Menstruation is resumed 28 to 40 days after cessation of treatment with a change to the normal picture in the smears, vaginal epithelium, and hormonal pattern.

In the menopause, male hormone frequently relieves symptoms without altering the vaginal smear or epithelium. No evidence of an "estrogenic" effect was noted. The urinary gonadotropic assay often gave values above normal at the time that symptoms had disappeared.

Diphosphothyamin (Phosphorylated Vitamin $B_{1}$ ) the Catalyst for the Oxidation and Dismutation of Pyruvic Acid in Animal Tissues and Bacteria. By E. S. GuzMAN BARRoN and (by invitation) CARL M. LyMaN, Chicago, Ill.

According to recent investigations vitamin $B_{1}$ polyneuritis is due to failure of the brain tissue to oxidize pyruvic acid, an intermediary product in the metabolism of carbohydrates. 1 There appears in the blood of polyneuritic animals a steady increase of pyruvic acid as the polyneuritic syndrome develops. ${ }^{2}$ Diphosphothyamin (phosphorylated vitamin $B_{1}$ ) is one of the catalysts for the decarboxylation of pyruvic acid.8 The metabolism of pyruvic acid in animal tissues and bacterial cells depends mainly on the presence of diphosphothyamin, and on the oxygen tension. The oxygen tension determines the relation between oxidation and dismutation of pyruvic acid. Diphosphothyamin, in the presence of oxygen, acts as catalyst for the oxidation of pyruvic acid; in the absence of oxygen it acts as catalyst for the dismutation of pyruvic acid. This catalytic action has been found in experiments with tissues of avitaminotic pigeons, with avian erythrocytes, and bacteria (gonococcus, Staphylococcus albus, hemolytic streptococcus). To act as catalyst, thyamin must first be phosphorylated. The phosphorylation rate of thyamin by tissues is fast; as a consequence there is no difference in the rates of oxidation of pyruvic acid by avitaminotic tissues whether thyamin or diphosphothyamin is used as catalyst. Some bacteria are unable to phosphorylate thyamin (gonococci, hemolytic streptococci). In these bacteria the addition of thyamin has no effect on the rate of oxidation of pyruvic acid even after an incubation of four hours at

\footnotetext{
1 Peters, R. A., Lancet, 1936, 1, 1161.

2 de Jong, S., Nederland. Tijdsch. Geneeskunde, 1937, 81, 935.

${ }^{8}$ Lohmann, K., and Schuster, Ph., Bioch. Ztschr., 1937, 294, 188.
} 
$38^{\circ}$; on the other hand, addition of diphosphothyamin increases the rate of this oxidation. Other bacteria (Staphylococcus albus) do phosphorylate thyamin at a slow rate. The relation between oxidation and dismutation of pyruvic acid, as expressed by the $\mathrm{O}_{2}$ uptake in air

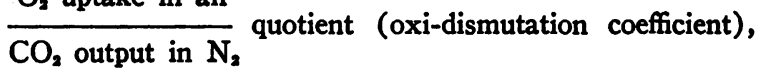
varies in different tissues and cells, being in general high in animal tissues and low in bacteria. The oxidismutation coefficient is highest in liver, being 3.65 ; in brain it is 3.22 ; in kidney, 3.06 ; in hemolytic streptococci, 0.85 . In avian erythrocytes there is oxidation of pyruvic acid but no dismutation. Prebluda and McCollum's reagent 4 (p-aminoacetophenone and nitrous acid), which gives a purple color with thyamin does not give this color reaction with diphosphothyamin. The catalytic power of thyamin disappears on reduction by colloidal platinum; this reduced thyamin is also unable to improve the condition of avitaminotic pigeons. Thyamin reduced by hydrosulphyte can be reoxidized by atmospheric oxygen with hystidine-hemochromogen as catalyst. These experiments suggest the opinion that thyamin acts as catalyst by being a reversible oxidation-reduction system.

Scurvy in the Guinea Pig. The Development of Anemia on a Diet Deficient in Vitamin C. By Stacy R. METTIER, San Francisco, Cal.

Two groups of young adult guinea pigs were subjected to a diet deficient in vitamin C. The diet of one group was supplemented with iron. In the first group, after a period varying from 14 to 30 days, signs of scurvy appeared accompanied by the development of anemia. In the iron-fed pigs, there was a lapse of from 20 to 40 days before scurvy and anemia appeared. However, the anemia in both groups was similar. There was a decrease of approximately $1,000,000$ red blood cells per cu. mm., and a loss of 2.75 grams of hemoglobin. The hematocrit values and indices showed the anemia to be hypochromic and microcytic in character. As the anemia progressed, there was a coincidental rise in the reticulocytes. A slight increase in the number of platelets was noted, but the leukocyte counts showed normal values. A group of animals with manifest scurvy and anemia were given orange juice daily, and this was followed by a return of the blood to normal and a disappearance of signs of scurvy.

The Relation Between Vitamin $B_{0}$ and the Unsaturated Fatty Acid Factor. By Thomas Whilam Birch (introduced by Joseph T. Wearn), Cleveland, Ohio.

By feeding rats on a fat free diet supplemented by graded amounts of vitamin $B_{6}$ it has been found that the animals will develop the typical acrodynia-like dermatitis ascribed to vitamin $B_{6}$ deficiency even though they are receiving adequate amounts of the vitamin. The acro-

4 Prebluda, H. J., and McCollum, E. V., J. Biol. Chem. (Proc.), 1937, 8, 1xxix. dynia produced by this means is curable by feeding a few drops of fatty acids prepared from corn oil.

When graded amounts of fat are fed to animals on a vitamin $B_{c}$ free diet it is found that the fat tends to delay the onset of the acrodynia and, moreover, when this symptom is developed it is not so severe.

These results indicate that two factors are concerned in the cure of the acrodynia-like dermatitis, one being the water soluble basic substance vitamin $B_{e}$, the other being fat soluble. The fat soluble substance appears to be similar to the unsaturated fatty acid factor of Burr and Burr and to the fat soluble antidermatitis factor of Hogan and Richardson.

These results would suggest that the physiological function of vitamin $B_{6}$ is connected in some way with the metabolism of the unsaturated fatty acids.

The Relation of Urinary Excretion of Vitamin $C$ to its Distribution in Blood. By MarTin HeINEMANN (introduced by John P. Peters), Boston, Mass.

When oral doses of 150 to $200 \mathrm{mgm}$. of cevitamic acid are taken by a subject saturated with this vitamin, there may occur a biphasic urinary excretion. The first peak occurs about 2 to 3 hours after ingestion and coincides with the highest serum level of cevitamic acid; the second coincides with the highest concentration attained in the red corpuscles. While the second phase of increasing urinary excretion is occurring, the serum concentration is falling but the red cell content is still rising.

Except during absorption of large doses of cevitamic acid, concentration of this vitamin is higher in erythrocytes than in serum.

Analyses of whole blood give a more reliable indication of the saturation of the body with vitamin $C$ than do analyses of either serum or red cells.

The Requirements for Ascorbic Acid by Patients with Peptic Ulcer. By Edward S. Emery, Jr., and (by invitation) Harry A. Warren and Michel Pijoan, Boston, Mass.

Numerous investigators have reported low plasma ascorbic acid values in patients with duodenal ulcer. Because plasma values alone do not give an index to the degree of tissue saturation or to the requirements of the body for this vitamin, it is the purpose of this communication to present a study of the ascorbic acid metabolism of such patients. As yet, the only systematic procedure to investigate this problem is the method described by van Eekelen and Heinemann, by which patients are saturated with vitamin $\mathrm{C}$ and a maintenance dose established for saturation. This procedure enables one to determine whether such patients require more ascorbic acid than normal subjects.

From our study, we have found that the tissues of these patients contained less ascorbic acid than the normal, and that these patients require somewhat more ascorbic acid per kilogram of body weight to keep them saturated. 
The Effect of Nicotinic Acid on Pellagra and Experimental Canine Black Tongue. By Julian M. Ruffin, G. Margolis, L. H. Margolis, and Susan Gower Smrth (by invitation) and David T. Smith, Durham, N. C.

Experimental canine black tongue of the Goldberger type has been produced in dogs and treated with varying doses of nicotinic acid. More than 50 tests have been made. The dogs responded as well to nicotinic acid as to liver for the first and second consecutive treatments but not to subsequent treatments. In primary and secondary attacks a daily intramuscular dose of $1 \mathrm{mgm}$. of nicotinic acid per kgm. for 10 days caused a satisfactory remission. Daily oral doses of $1.5 \mathrm{mgm}$. per $\mathrm{kgm}$. for 10 days were as effective as the same dose administered parenterally. Increasing the oral daily dose to $10 \mathrm{mgm}$. per $\mathrm{kgm}$. gave no better results.

Five patients with acute pellagra have been treated by daily intravenous and intramuscular doses of $1.5 \mathrm{mgm}$. of nicotinic acid per $\mathrm{kgm}$. of body weight. The mental symptoms improved even more rapidly than with liver therapy. The mucous membranes showed improvement in 24 hours, and the tongue usually had a normal color in 48 hours. The diarrhea rapidly subsided and the appetite markedly improved in 4 to 5 days.

However, nicotinic acid therapy required twice as long as liver treatment for the healing of the typical dermatitis of the hands and feet and of the seborrhea of the face.

After nicotinic acid treatment, 4 of the 5 patients were exposed to radiant heat from the sun or from a heater, and none relapsed.

All 5 cases of pellagra were cured with nicotinic acid, although the dermatitis improved more slowly than with liver treatment.

These few observations on the differences in the rate of improvement of the mental, mucous membrane, and dermal signs seem to indicate that there are two or more factors in liver concerned in the rapid cure of pellagra.

Evaluation of the Intestinal Factor in the Increase of Blood Urea Nitrogen Following Massive Hemorrhage from the Stomach and Duodenum. By LEON ScHIFF and (by invitation) SANDER Goodman and William Bennett Bean, Cincinnati, Ohio.

The blood urea nitrogen is frequently elevated following massive hemorrhage from the upper digestive tract. This elevation is of prognostic significance. It is not associated with change in blood chlorides or carbon dioxide combining power of the blood.

Various factors have been invoked to explain this increase of urea, such as dehydration, shock, increased tissue breakdown, renal failure, and absorption of products of decomposition of the blood in the intestinal tract. In an attempt to evaluate the importance of the intestinal factor, between 750 and $2,000 \mathrm{cc}$. of citrated human blood were given by stomach tube to a group of controls and to patients who had had a previous hematemesis with elevation of blood urea nitrogen. Urinary and blood urea nitrogen determinations were made before and at frequent intervals after the administration of the blood.

The Effect of Acetyl B Methyl Choline (Mecholyl) on Gastric and Hepatic Secretion. By James FlexNer (by invitation) and IRving S. WRIGHT, New York, N. Y.

In humans $25 \mathrm{mgm}$. of mecholyl subcutaneously produced an actual increase in free and total acidity over controls. Erroneous conclusions have been previously drawn by other workers regarding this, since unless care is exercised the gastric contents become mucinous and alkaline due to swallowing of the great excess of saliva produced. Amylase and total salivary determinations demonstrated that this was due to saliva. Rabbit and cat experiments tended to confirm this.

In cats mecholyl subcutaneously and intravenously produced a slight increase in hepatic secretion followed by a lag. Mecholyl injected into a peripheral vein produced a fall in arterial and portal pressure which was sometimes followed by a rise in portal pressure. Mecholyl injected into the portal vein produced a lesser fall in arterial pressure and a rise in portal pressure without a previous fall. When the dosage was adequate to cause a sharp arterial pressure drop the effect on the liver secretion was constant regardless of the route of the injection. These observations may explain in part why oral administration of mecholyl produces a much less severe effect on blood pressure and peripheral circulation than by other routes. Histamine caused similar effects in hepatic secretion and arterial pressure.

Deficiency Syndromes Secondary to Short Circuited Small Intestine. By MADELAINE R. Brown (introduced by Tracy J. Putnam), Boston, Mass.

The case histories of four patients with accidental short circuiting of the major portion of the small intestine are outlined from the recent literature. In at least two of these patients chronic diarrhea set in immediately following operation. Two developed degeneration of the peripheral nerves and spinal cord, while in two a macrocytic anemia supervened. Was the deficiency state due to interference with absorption area or to interference with normal motility of the gastro-intestinal tract?

In an effort to answer this question, two sets of animal experiments were performed. Almost all, or part of the small intestine was removed in three pigs, in three others almost all, or part of the small intestine was short circuited and the entrance to the loop partially tied off. Removal alone did not lead to diarrhea, anemia or paralysis. Partial short circuiting had no significant effect on peristalsis or the blood picture, but short circuiting of almost the entire small intestine was followed by diarrhea and severe microcytic anemia.

Abnormalities of peristalsis interfering with both digestion and absorption apparently constitute a more important factor in the production of deficiency syndromes than reduction of absorption area alone. 
Availability of Carbohydrate of Various Foods and Relationship to Use of Protamine Zinc Insulin. By HERberT Pollack and (by invitation) Henry Dolger, New York, N. Y.

Fifty-five diabetic patients using protamine zinc insulin were given equi-carbohydrate amount of orange juice, ripe banana, ripe banana plus cream, and meat. These foods were taken on an empty stomach twentyfour hours after the last dose of protamine zinc insulin. Blood sugar estimations were made at half-hour intervals for four hours.

The results show that orange juice is rapidly absorbed, producing a hyperglycemia in thirty minutes to one hour. This rise is followed by a precipitous drop, frequently to hypoglycemic levels. Ripe bananas are absorbed on the whole more slowly, but not with regularity. The addition of cream to the banana delayed peak absorption to the end of the second hour. There were no precipitous drops in blood sugar following the banana and cream meal. Meat, as previously shown by Conn and Newburgh, caused very little change in blood sugar.

The practical application of this lies in the selection of foods for diabetics using protamine zinc insulin. Avoidance of fruit juices prevents transient glycosuria and shock. Use of fat, as cream, prolongs absorption and prevents wide fluctuation in blood sugar levels. The carbohydrate equivalent of meat given at supper time will be available to the body during the long interval until breakfast, preventing nocturnal hypoglycemia. These diet principles have been applied clinically to one hundred and fifty patients for eighteen months.

The Response to Insulin in Diabetes Mellitus and in Experimental Pituitary Diabetes. By F. C. DoHAN (by invitation) and F. D. W. Lukens, Philadelphia, Pa.

The response to insulin has been employed as a means of separating diabetics into two types which have been designated "insulin sensitive" and "insulin insensitive." It has also been reported that serum from insulin insensitive diabetics when injected into rabbits impairs the animals' response to insulin. Both of these procedures have been tried in the present study.

As far as an individual patient is concerned the insulin test may vary considerably at different times. The reason for this variation in response is not clear and may obviously depend on several factors. No significant alteration in the insulin curves was found in our series of rabbits tested before and after the injection of diabetic serum.

On the other hand, pituitary extract injections in normal animals may completely prevent the response of the blood sugar to insulin. This has been found both in acute experiments in rabbits and in chronically injected dogs. The difference in the response to insulin in clinical diabetes and in "pituitary diabetes" will be considered in comparing the two conditions.
Carbohydrate Tolerance after Protamine Insulin: Its Bearing on the Physiology of Insulin Secretion. By HeNRY T. RicketTs (by invitation) and Walter L. Palmer, Chicago, Ill.

There is considerable evidence favoring the theory that in the normal individual the ingestion of carbohydrate stimulates the secretion of insulin by the pancreas.

Soskin and his coworkers, however, in 1934, showed that in the depancreatized dog receiving a constant intravenous injection of glucose and insulin so adjusted as to maintain the blood sugar normal and level, an added dose of glucose gave a normal sugar tolerance curve provided the liver was intact. Extra insulin therefore was neither available nor required.

Protamine insulin has now provided a means of investigating this question under more physiological conditions. It has been demonstrated in severely diabetic patients and totally depancreatized dogs that a dose of protamine insulin, given on one day, which is adequate to maintain a normoglycemic plateau during the entire ensuing morning without food is not adequate to prevent hyperglycemia after a moderate or even low carbohydrate breakfast under otherwise identical conditions. Mild diabetics, on the other hand, respond to such a meal after protamine insulin with an almost normal blood sugar curve.

Thus in the severely diabetic organism postprandial hyperglycemia is not controlled without extra insulin. It is possible that the mild diabetic has enough functioning pancreas to respond to the ingestion of food by secreting the required extra insulin.

These observations support, though they do not prove, the theory that normally hyperglycemia constitutes at least one factor in the regulation of insulin secretion.

\section{The Rôle of Potassium in the Mechanism of Family}

Periodic Paralysis. By RoBert H. Pudenz (by invitation) and JoHN F. McIntosh and Donald MCEAchern, Montreal, Canada.

We have been able to demonstrate in a patient studied by us that attacks of paralysis are associated with a marked fall of serum potassium and that administration of potassium results in rapid clearing of all symptoms. Since our first observation the carefully studied case of Aitken et al 1 has appeared and our results are entirely confirmatory.

The patient, a male of 18 has had recurrent attacks since the age of 11 . They occur about once a week at present and last 24 to 48 hours. The father, brother, and sister have been similarly affected. The attacks are typical. They come on without apparent relation to diet, exercise, etc., and usually at night. In severe attacks, paralysis of the muscles of limbs and trunks is complete; the respiratory muscles are little affected and the bulbar and facial muscles scarcely at all. Reflexes and response to galvanic and faradic stimulation are absent. There

1 Aitken, R. S., Allott, E. N., Castleden, L. I., and Walker, M., Clin. Sc., 1937, 3, 47. 
is a fall of heart rate to 40 , dilatation of the pupils, and a subnormal temperature. Between attacks he is entirely normal.

In intervals between attacks serum potassium has been found to be 18 to $21 \mathrm{mgm}$. per cent; during attacks it falls to levels of about $10 \mathrm{mgm}$. per cent, the lowest recorded being $8.6 \mathrm{mgm}$. per cent. The threshold for paralysis appears to be about $10 \mathrm{mgm}$. per cent. Administration of $\mathrm{KCl}$ in large doses ( 5 to 15 grams) by mouth brings about disappearance of paralysis within 5 to 7 hours. When given intravenously muscle power begins to return within 5 minutes and recovery is practically complete within 30 minutes. Reflexes, electrical excitability, etc., return simultaneously. Injections of from 0.5 to 2.0 grams, may bring about recovery but blood taken during partial recovery shows no increase of potassium indicating that the potassium is going elsewhere, probably the muscles. Carbon dioxide combining power is lowered during attacks from about 64 volumes per cent to about 50 volumes per cent, but studies of sodium, chlorides, calcium, and protein have revealed no significant abnormality. There are wide variations of urinary creatinine output and a consistent output of creatine (up to 0.3 gram per day) both during and after attacks and in the intervals.

Prostigmine causes slight temporary improvement during attacks but nothing comparable to the complete relief caused by potassium. Ergotamine tartrate is without effect. Atropine results in marked increase of heart rate during the bradycardia of attacks but no benefit to muscle power. Adrenalin has no effect on muscle power and the normal rise in blood potassium due to it is lacking. Attempts to produce attacks by exercise and exposure to cold have failed. Large doses of glucose by mouth, sometimes combined with insulin injections following the technique of the English workers, have apparently produced attacks on two occasions but have failed on two. The level of acetylcholine esterase in corpuscles and serum has not been abnormal and no acetylcholine has been found in the blood.

The patient is at present on a balance experiment to determine the fate of potassium and results will be reportable soon. It seems apparent that this condition must be classed with others as an inborn error of metabolism.

\section{The Demonstration by Liver Function Tests and Histo-} pathological Studies of the Interrelationship of Liver and Gallbladder Disease. By MYER TEITELBAUM (by invitation), Arthur C. Curtis and S. Milton GoldhaMer, Ann Arbor, Mich.

Several liver function studies were performed simultaneously on thirty-eight patients to determine the constancy and degree of impairment of liver function associated with cholecystitis. The tests employed were the urinary urobilinogen, mean corpuscular volume, and serum protein determinations; the Takata-Ara and bromsulphalein dye excretion tests; the galactose and glucose tolerance tests; and the van den Bergh reaction. Histopathological studies of the liver were made on eleven pa- tients of this group. Gross operative observations of the liver were available in sixteen patients. Eleven patients with cholecystitis were not operated upon.

There was an extremely high percentage of positive tests in the patients demonstrating histological and gross anatomic hepatic pathology. A lesser percentage of positive tests was found in those patients demonstrating no gross liver pathology.

Liver function tests and histopathological studies of the liver show that hepatic damage of varying degree is a common accompaniment of cholecystitis. In many cases the severity of liver pathology, proven microscopically, is clearly indicated by the liver function tests. The proper use and interpretation of these tests should be of great value in the choice of proper treatment and in prognosticating the course of the disease.

The Relationship of the Liver to the Utilization of Intravenously Injected Sodium d-Lactate. By L. J. SofFer, D. Alfred Dantes, and Harry Sobotra (introduced by George Baehr), New York, N. Y.

The object of these experiments was to study the utilization of intravenously injected sodium $d$-lactate by human beings both under normal conditions and in the presence of certain pathologic processes. The essential differences between the metabolism of the $d$ and $l$ forms of lactic acid as was pointed out by Meyerhof and Lohmann, Cori and Cori, and Abramson, Eggleton, and Eggleton, consist in the fact that the liver is concerned with the utilization of the $d$-salt, but not of the $l$-salt. Cori and Cori found that while 40 to 95 per cent of injected $d$-lactate in the rat is converted into liver glycogen, 30 per cent of the injected $l$-salt is excreted in the urine, but the remainder is converted into $\mathrm{CO}_{2}$ without any conversion into glycogen by the liver.

In the present series of experiments, sodium $d$-lactate in a dosage of $75 \mathrm{mgm}$. per $\mathrm{kgm}$. of body weight of a 12 to 14 per cent solution was injected intravenously in 76 instances. The patients investigated were 25 normal individuals, 25 instances of acute diffuse hepatic parenchymal injury with icterus, 15 instances of extrahepatic obstructive jaundice, 8 cases with various muscular dystrophies and in 3 instances with diabetes. It was found that in the normal instances the increase in the lactic acid content above the control value following the injection of sodium $d$-lactate varied from 15.6 to $41.2 \mathrm{mgm}$. per cent. This peak was reached within five minutes. After the peak, the lactic acid content of the blood fell rapidly, the major drop occurring within twenty minutes and more gradually thereafter, returning approximately to the control level within thirty minutes. No increase in the urinary excretion of lactic acid was manifested. In contrast to this group, the patients with acute diffuse hepatic parenchymal damage with icterus showed a considerable delay in the disappearance of the injected $d$ lactate, so that at the end of one-half hour the lactic acid blood level showed a considerable elevation above the control level, the elevation varying from 30 to 164 per cent. The patients with extrahepatic obstructive jaun- 
dice, muscular dystrophies, and diabetes mellitus with and without insulin behaved as did the normal controls.

The results obtained suggested the use of intravenously injected sodium $d$-lactate as a test for the differentiation between icterus due to hepatic parenchymal injury and extrahepatic obstruction.

For purposes of comparison, it was decided to perform several other liver function tests on the patients with jaundice. The other tests employed were the cholesterolester determinations, the galactose tolerance test, the urinary excretion of urobilin and the sodium-benzoate test. It was found that in the patients with acute hepatic parenchymal injury the $d$-lactate clearance test yielded the largest incidence of positive results, while. in the patients with extrahepatic obstructive jaundice, this test was uniformly negative. The diagnosis in the latter group of patients was confirmed in all instances either by operation or necropsy.

In view of the results obtained, it is suggested that the sodium $d$-lactate blood clearance may be of value in the differentiation in instances of jaundice, between extrahepatic obstruction, and diffuse hepatic parenchymal damage.

The Osmotic Activity of the Proteins of the Blood in Chronic Atrophy of the Liver and in Obstructive Jaundice. By HugH R. BuTt (by invitation) and Albert M. SNell, Rochester, Minn.

Hypoproteinemia and reversal of the albumin-globulin ratio is a fairly constant finding in parenchymatous disease of the liver, but few direct measurements of the colloid osmotic pressure in such cases have been reported. Using a modification of the Starling-Adair membrane method, the colloid osmotic pressure of normal serum has been found to be approximately $371.3 \pm 5.38 \mathrm{~mm}$. of water. In individuals with chronic atrophy of the liver (cirrhosis), the colloid osmotic pressure of the serum often was reduced to half the normal value. In some cases in which edema and ascites were present, the value for colloid osmotic pressure was lower than the estimated hydrostatic pressure of the portal venous system, as calculated by McIndoe (1928) and by Thompson and others (1937). In individuals with obstructive jaundice the values for colloid osmotic pressure also were substantially reduced (200 to $275 \mathrm{~mm}$. of water), often without respect to the depth of jaundice in the individual case.

In all types of cases studied there was no constant relation between the level of serum protein and the colloid osmotic pressure; there was, as might have been predicted, a better correlation between osmotic pressure and serum albumin content. In hepatic disease no constant "edema level" as measured by the above-mentioned procedures could be demonstrated.

A Study of the Stercobilin Tolerance of the Liver under Normal and Pathological Conditions. By CeCIL JAMES Watson, Minneapolis, Minn.

Urobilinogen has recently been shown to be a composite of two chromogens, i.e., mesobilirubinogen and stercobilinogen. The latter of the two is the mother substance of stercobilin, which may be isolated in crystalline form from human feces (in largest yields from cases of hemolytic jaundice). It is this substance which has been used in the present study.

Urobilinogen is usually increased in the urine in the presence of diffuse affections of the liver. The value of simply estimating the 24-hour amount is considerable, but is somewhat reduced because of factors which may cause a diminished formation or absorption of urobilinogen. These factors are discussed briefly.

The purpose of the present study has been to supply the liver with a fixed amount of stercobilin in a short space of time in order to determine its ability to dispose of it. Evidence is presented which reveals that the normal liver rapidly excretes the amount injected into the bile. In the present study the "stercobilin tolerance" of the liver was studied in 70 individuals, 20 of whom served as controls. In the latter, the fraction appearing in the urine was small, not exceeding $5.0 \mathrm{mgm}$., after the intravenous injection of $50 \mathrm{mgm}$. of crystalline stercobilin. In patients with various types of jaundice and liver disease the fraction excreted in the urine exceeded $5.0 \mathrm{mgm}$. in most instances, values ranging from 5 to $49 \mathrm{mgm}$.

Of considerable interest is the fact that the injected stercobilin is regularly reduced, at least in part, to stercobilinogen, so that the Ehrlich reaction of the urine becomes positive soon after administration of the substance. The actual site of reduction remains to be determined.

In the presence of renal insufficiency the output of stercobilin and stercobilinogen in the urine of patients with coexisting liver dysfunction is markedly slowed, so that it often requires two or three days before the urinary value returns to the previous level.

The Influence of Fat upon the Excretion of Urobilin. By Hugh W. Josephs, L. Emmett Holt, JR., and (by invitation) Herbert C. Tidwell and Charlotte $N$. KAJDr, Baltimore, Md.

The view that ingested lipids were responsible for certain types of hemolytic anemia (bothriocephalus anemia, goat's milk anemia) has received little attention in recent years. Other explanations of these anemias have come to the fore, and attempts to produce anemia in experimental animals and in man with diets high in fat have met with little success. These negative results do not, however, eliminate the possibility that fats exert some hemolytic action. It seems possible that mild degrees of hemolysis might be produced, insufficient under ordinary conditions to lead to anemia.

We attempted to test out this possibility by measuring total urobilin excretion in a series of normal infants who were fed on diets low and high in fat. Somewhat to our surprise it was found that when 80 per cent or more of the calories were supplied in the form of fat, a rise 
in urobilin output was regularly obtained; in no instance, however, did anemia or reticulocytosis develop. Observations were made with a number of different fats: cow's milk fat, goat's milk fat, soya bean oil, olive oil, corn oil, human body fat and an odd carbon fat consisting of a mixture of saturated glycerides with 15 and 17 carbon atoms. No difference was demonstrated in the effect of these different fats. When free fatty acid or soap was added to the formula a smaller quantity sufficed to produce the increased urobilin output than was the case with neutral fat.

Attempts were made to find an explanation of the rise in urobilin output on a high fat diet. It was found that the phenomenon could not be attributed to ketosis, since the addition of a small amount of sugar, sufficient to abolish the ketosis, failed to reduce the urobilin output. Another possibility was that fat increased the flow of bile by emptying the gallbladder and that no actual increase in the production of bile pigment occurred. If such an explanation were correct, one would expect the effect to be a temporary one; we found, however, that the high output of urobilin was not diminishing at the end of two weeks. Still another possibility was that the increased amount of fat in the intestine served as a solvent for bile pigment and thus brought about its loss from the body. To test this we carried out experiments in which mineral oil was added to the diet. This should duplicate any solvent effect on bile pigment which high fat would have. We did indeed find a temporary increase in urobilin output when mineral oil was added to the diet, but this was not maintained, the urobilin falling to its original level within a few days. It thus appears that prevention of reabsorption of bile pigment will not explain the increased excretion on a high fat diet.

By exclusion therefore we are inclined to conclude that the increased urobilin output on a high fat diet is due to increased blood destruction of such a low degree, however, that it is not of clinical significance in the normal individual. Whether it is of significance in pathological states remains to be determined.

\section{Electrolyte Balances during Artificial Fever with Special} Reference to Loss through the Skin. By E. HENRY Keutmann (by invitation), and SAmuez H. BassetT and Stafrord L. Warren, Rochester, N. Y.

Electrolyte balances were determined on four patients before, during, and after the induction of artificial fever by radiant energy. Losses through the skin were measured directly during fever and during a control day with no visible sweating.

During the control days skin loss of sodium varied from 0.069 to $0.237 \mathrm{gram}$, chlorine loss from 0.083 to 0.281 gram.

The duration of fever and the accompanying losses of electrolytes through the skin were as follows:

\begin{tabular}{|c|c|c|c|}
\hline \multirow{2}{*}{ Fever } & \multicolumn{3}{|c|}{ Skin loss in m.eq. } \\
\hline & $\mathrm{Na}$ & $\mathbf{C l}$ & $\mathbf{K}$ \\
\hline 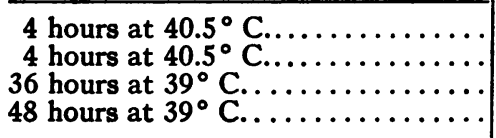 & $\begin{array}{r}130 \\
109 \\
331 \\
83\end{array}$ & $\begin{array}{r}154 \\
99 \\
266 \\
71\end{array}$ & $\begin{array}{r}9 \\
18 \\
52 \\
29\end{array}$ \\
\hline
\end{tabular}

The skin losses of sodium and chloride represented from seven to nineteen per cent and the net losses (negative balances) from seven to twenty-two per cent of the amount estimated to be present in the extracellular fluids at the beginning of treatment.

Differences in the losses from the skin were dependent upon individual variation. The excess of sodium over chloride in the sweat of three patients and the reverse condition in the other patient is evidence against the secretion of definite proportions of these elements with one another by the sweat glands.

At the end of fever the viscosity, hematocrit, serum solids, and serum proteins in the blood of all the patients were found to be increased. In three of the patients the concentrations of sodium, chloride, and carbon dioxide of the serum water were decreased, in one they were increased.

The two patients who developed the greater dilution of electrolytes in the serum had symptoms characteristic of heat cramps.

In three patients the loss of weight was less than that calculated from the electrolyte loss, in the fourth the loss of weight was greater than that calculated from the electrolyte loss. This seeming discrepancy may be explained by progressive dilution or concentration of the electrolytes remaining in the body.

Three to four days were required to replace the sodium and chloride deficit. In each case the retention after fever was slightly greater than the amount lost.

Iron Excretion: Histological Study by Means of Grafts of the Colon upon the Abdominal Wall. By STEPHEN J. Maddock (by invitation) and Clark W. Heath, Boston, Mass.

A section of the sigmoid of four dogs was carried to the abdominal wall with its blood supply and successfully grafted. Repeated biopsies could be made upon these grafts with ease. Such preparations prevented exposure of intestinal surface to iron-containing intestinal contents.

Iron was injected parenterally daily or given daily by mouth to these dogs and also to four normal dogs. Biopsies of the graft and of various parts of the gastrointestinal tract and other organs were made before and after the administration of iron. Sections of tissue were stained for iron by the potassium ferrocyanide method, using careful precautions against iron contamination.

There was no definite increase of iron in the wall of the graft or of the stomach, duodenum, ileum, cecum, and colon in situ. Iron was present in greatly increased quan- 
tity in the liver and spleen, and to a lesser extent in the kidney and skin after the administration of large amounts of iron.

The absence of demonstrable increase of iron in the gastro-intestinal tract under the conditions of this experiment fails to show that the large intestine is the site of iron excretion, and corroborates the recent findings of other workers in the field of iron metabolism, namely, that iron is not excreted to any appreciable degree by the intestinal tract.

The Relationship between the Fractions of the Serum Protein Complex. By Henry FIELD, JR., and (by invitation) Daniel Melnick and Christopher ParNall, Jr., Ann Arbor, Mich.

There has been produced much evidence to indicate that the serum protein complex is composed of two or more unstable coprecipitation systems in mutual equilibrium and that the protein fractions isolated by physicochemical methods are not preexistent. Variations in the concentrations of the isolated fractions are considered to result from disturbances in the balance of the component systems.

We have dialyzed normal sera against nephrotic sera, characterized by hypoproteinemia and markedly low albumin to globulin ratios, for variable periods of time and at selected temperatures. In this manner the normal and nephrotic serum proteins were subjected to practically the same environmental influence with respect to concentration of protein and of the crystalloids, $\mathrm{pH}$, and temperature. Nevertheless, no change in the albumin to globulin ratios of either the normal or nephrtoic sera resulted. Serum lipids were observed to exert no appreciable influence upon the salting out of the protein fractions.

These studies are not interpreted as indicating that such protein fractions are preexistent in serum. They seem to indicate that any association or dissociation of protein molecules must occur within independent systems and that fractionation obtained by precipitation methods is different from that obtained by ultracentrifugation. The reason for the change in ratio of precipitation fractions in pathological states remains to be explained.

\section{A Long Term Study of the Variation of Serum Choles-} terol in a Group of Relatively Normal Individuals. By KenNeth B. Turner and (by invitation) Alfred StEINER, New York, N. Y.

Although there are innumerable reports on the blood cholesterol of man under normal or abnormal conditions, the vast majority of these are based on single or, at best, a few determinations in a given individual. Little is known of the normal variation from week to week over long periods under controlled conditions.

An unusual opportunity was afforded to study the serum cholesterol of 11 relatively normal but hospitalized patients at approximately weekly intervals for a year or more. Frequent determinations of the basal metabolic rate and other factors that might influence the serum cholesterol were made.

The constancy for the individual of the cholesterol level in his serum was established. Thyroid extract produced a marked fall in the serum cholesterol. High or low fat diets, the administration of cholesterol or of potassium iodide were without effect.

Furthermore, no significant variations in serum cholesterol occurred during the course of a day. The futility of the so-called "cholesterol tolerance tests" was demonstrated.

Allergic Bronchial Obstruction and Bronchiectasis. By Herman H. Riecker, Ann Arbor, Mich.

Bronchiectasis has been due, in occasional cases, to extrinsic pressure on a small bronchus collapsing its lumen, to cancer, or to fibrosing and inflammatory lesions which obstruct the bronchus. The common factor in these cases is an obstruction with trapping of the secretion and consequent infection.

In searching for a cause more applicable to the large majority of cases, it seemed advisable to investigate the possible relationship of bronchiectasis to allergic disease of the respiratory tract.

Pathological studies, particularly by Alexander, have shown that in asthma, bronchial obstruction does occur at about the $5 \mathrm{~mm}$. level, and recent lipiodal studies here confirm this finding for the active case. It is not a diffuse process and is produced mostly by edema and mucus. Below this level there often is dilatation in the walls of the bronchioles with the occasional formation of bullae and emphysema. Eosinophilic infiltration of muscle and degeneration of cartilage are observed. In autopsy material of bronchiectasis, eosinophiles in the wall of the bronchus above the infected area have been seen, and it has not been uncommon to find many eosinophiles in the bronchiectatic sputum when proper search is made.

The hospital records of 122 consecutive cases, diagnosed as bronchiectasis by bronchograms, and containing the results of an examination of the nasal membrane by competent otologists, were studied with respect to the presence of allergy, with the following results :

\section{Cases of bronchiectasis with}

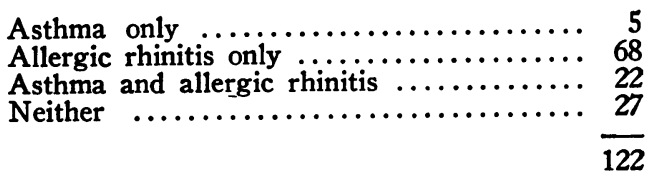

Thus 77.8 per cent of the cases of bronchiectasis appear to have occurred in individuals with allergic respiratory tract disease which long antedated the symptoms of bronchiectasis. In the remaining cases there was no proof of allergy, possibly because in some cases the allergic changes were reversible and not constantly present in the nasal membrane.

Bronchoscopic observations in our clinic have shown that in allergic rhinitis, and in many cases of bronchiectasis, the allergic edema is by no means limited to the 
nasal membrane but may extend quite far down the individual bronchi without producing the symptom of asthma. In bronchiectasis, complete obstruction of individual bronchi of the third order has been seen to disappear after the application of adrenalin and cocaine. Similarly, it has been observed that in asthma the nasal membrane frequently is involved in the allergic process.

In view of these observations, it is not unreasonable to suppose that the allergic bronchial reaction associated with allergic rhinitis produces partial or complete obstruction with distal dilatation and weakening of the bronchial wall to form the basis for many cases of bronchiectasis. The purulent upper respiratory infection (present in 72 per cent of the cases) may be a contributing or activating factor but not a primary one. This conception of the basic etiology of the disease offers some hope for its anticipation and prevention in the allergic child. In the treatment of the disease by drainage, the factor of edematous obstruction may well be taken into consideration.

Cytological studies for eosinophiles in material obtained by bronchoscope from the bronchiectatic cases are being continued.

\section{Studies in Tuberculous Calcification. By RoBerT G.} Bloch, Chicago, Ill.

Calcification, besides resorption, is the only healing process of the tuberculous lesion itself. The deposition of calcium salts sufficient to be clinically recognized as calcification is a very slow process. The clinical conception of calcification is based exclusively on its roentgenological appearance, the degree of density being the chief criterion. The grossly calcareous lesion represents the desired final stage of healing and is erroneously confused with the degree of calcification, i.e., with the amount of calcium deposited. While the amount of calcium expresses itself roentgenologically, the calcareousness does not. Involvements with a very high calcium content may still be softly caseous and are potential excavations of clinical importance. The size of a lesion is a better diagnostic guide than the roentgenologic degree of density.

Small tuberculous lesions in the lung, with certain characteristics as to size, location, consistency, chemical content, and roentgenological appearance, are universally accepted as evidence of primary "childhood" infection. From the studies of Ghon in children, the conclusion was reached, without sufficient evidence, that such lesions, when found in adults, signify the remains of an infection contracted in early life.

Without making a definite contention that the existing conception is erroneous, the question is opened in this paper if many of the so-called primary tuberculous foci do not develop during adult life. Some clinical evidence is presented tending to show that they may be another form of tuberculosis developing in adults as the result of exogenous superinfection. The initial results of an experimental study comparing roentgenological, histological, and chemical factors are discussed.
Induction of Lymphoma by Carcinogenic Agents. By Austin M. Brues and (by invitation) Beula $B$. Marble, Boston, Mass.

A study has been made of the effect of cancer-producing agents on animals with a constant low susceptibility to lymphoma. One hundred and eighty mice were used, of a special strain in which the normal incidence of lymphoma is approximately 2 per cent, and the incidence was markedly increased by the application of carcinogenic tar to the skin. Three batches of tar were used, of widely varying degrees of carcinogenic potency, and the incidence of lymphoma followed, pari passu, the potency of the tar, reaching 50 per cent in the group receiving the most actively carcinogenic agent. The lesions produced were characteristic, involving spleen, lymph nodes, and usually liver, with an associated subleukemic blood picture. The disease in control animals runs a relatively benign course of several months, while in tarred animals it progresses rapidly and they usually die within a few weeks of the clinical onset. Although cancer-producing agents do not readily produce lymphoma in other strains of animals, these results suggest that in the presence of a latent predisposition they may influence its development and course.

The Entrance of Proteins into Joints and Certain Other Body Cavities. By Granville A. Bennett and (by invitation) Morris F. Shaffer, Boston, Mass.

Previous studies have demonstrated that foreign proteins injected intra-articularly are removed from joints solely by way of the lymphatics, whereas drugs in aqueous solution are removed from joints chiefly by way of the blood capillaries. The purpose of the present experiments was to study the transference of proteins from the blood stream into joints. Information so obtained should lead to a better understanding of normal joint physiology and the mechanism involved in the production of joint effusions. In addition, the passage of such substances into the aqueous chambers and the subarachnoid space was investigated.

Crystalline egg albumen or horse serum protein fractions were injected intravenously into rabbits. By employing the precipitin test with specific antisera as a means for their detection, these proteins could be demonstrated to have passed regularly, within a short period of time, from the blood stream into the knee joints. They also appeared in the aqueous chambers of the eyes, but in lower concentration. In most experiments no foreign protein was detected in the spinal fluid; when present its concentration was considerably lower than in the joint washings or aqueous fluids.

Such data concerning the entrance into and residence in various body cavities of foreign proteins should serve as a basis for experimental studies on tissue hypersensitivity and the treatment of infections of joints with specific antisera. 
Unequal Distribution of Respiratory Gases in Emphysematous Subjects; its Measurement and Significance. By A. Cournand and J. S. Mansfteld (by invitation) and D. W. Richards, JR., New York, N. Y.

Several different techniques were employed, with normal and emphysematous subjects, to test the state of mixture of intrapulmonary gases, both before and during the course of quiet breathing in a small closed circuit consisting of lungs, spirometer, soda lime container, and connecting tubing. Varying factors in the technique were: 1, (a) preliminary breathing of pure oxygen for ten minutes before onset of rebreathing; or $(b)$ preliminary air breathing; 2, variation in composition of gases in spirometer before rebreathing; 3 , (a) steadily decreasing volume of closed breathing circuit, or (b) maintenance of constant volume of lung-spirometer circuit. With each of these methods it is possible, after certain corrections are made, to calculate pulmonary residual air values, according to the principle of nitrogen dilution.

In normal subjects all techniques gave essentially the same values for functional residual air.

In certain emphysematous subjects, washing out of the lungs by pure oxygen breathing, preliminary to the closed circuit breathing, gave residual air values much lower than the (usually large) values found after preliminary air breathing. This suggests that the resting emphysematous lung contains excess nitrogen, presumably in the large hypoventilated but still perfused pulmonary spaces.

The discrepancy between the residual air values obtained by these different preliminary breathing procedures can be used as a quantitative measure of unequal distribution of respiratory gases in pulmonary spaces. The large values found for residual air in emphysematous subjects are in some cases due in part to the same phenomenon. Such residual air figures in these cases may be considered as a combined index of physiological dysfunction, rather than as a measure of strictly anatomical volume.

The Inhibition of Choline Esterase of Muscle by Prostigmine with Reference to the Action of the Drug in Myasthenia Gravis. By William C. Stadie and (by invitation) MAxweLl Jones, Philadelphia, $\mathrm{Pa}$.

Recently prostigmine has been shown to ameliorate dramatically but briefly the symptoms of myasthenia gravis. In explanation, the hypothesis has been advanced in the literature that the disease is associated with a high content of muscle choline esterase. In consequence, acetylcholine, when formed, is destroyed so rapidly that the ordinary neuro-humoral mechanism of nerve-muscle transmission is impaired and symptoms of the disease result. According to this view, prostigmine produces its effect by partial inhibition of the esterase of the muscle thus restoring to normal the neuro-humoral mechanism.

No muscle esterase values in myasthenia cases have been reported and the serum esterase values are found to be within normal limits. However, the association of marked inhibition of serum esterase and the alleviation of the symptoms by prostigmine still lends color to the hypothesis.

We have studied the effect of prostigmine upon the esterase of serum and muscle of guinea pigs, normal humans, and in one case of myasthenia gravis. In all cases the results are essentially the same and may be summarized as follows :

In guinea pigs, the intravenous injection of eserine or prostigmine in doses which are comparable to therapeutic doses in humans resulted in marked inhibition of the choline esterase activity of the serum. In muscle, however, we were unable to show any inhibition whatever. Even with toxic doses no inhibition could be shown. The following is a typical protocol:

The choline esterase activity of muscle and serum of a guinea pig before and after the intravenous injection of $1.0 \mathrm{mgm}$. of eserine (activity in $\mu M$. per gram per minute of acetylcholine hydrolyzed)

\begin{tabular}{|c|c|}
\hline Muscle & Serum \\
\hline $\begin{array}{l}\text { Before . . . . . . . . . } 0.68 \\
\text { After . . . . . . . . . } 0.69\end{array}$ & $\begin{array}{l}0.66 \\
0.20\end{array}$ \\
\hline
\end{tabular}

When muscle or serum is equilibrated in vitro with known concentrations of prostigmine it was found that, whereas serum esterase is markedly inhibited, there was little or no inhibition of muscle esterase by prostigmine at concentrations within the therepeutic zone. Only when the concentration of the prostigmine greatly exceeded the therapeutic concentration could inhibition be demonstrated, and in all cases this was much less than in the case of the serum. The following is a typical protocol in the guinea pig.

\section{Choline esterase activity of guinea pig muscle and serum when exposed in vitro to known concentrations of prostigmine. Results are expressed as a ratio to the value in the absence of prostigmine}

Concentration
of prostigmine
mgm. per liter
0.0
0.05
0.1
0.5
1.0
2.0

\begin{tabular}{lcc} 
& \multicolumn{2}{c}{ Relative esterase activity } \\
& Muscle & Serum \\
Therapeutic zone & 1.00 & 1.00 \\
Therapeutic zone & 1.00 & 0.39 \\
& 0.90 & 0.39 \\
& 0.59 & 0.23 \\
& 0.32 & 0.07 \\
& 0.23 & 0.04
\end{tabular}

In one case of myasthenia gravis the choline esterase of the muscle (1.63 $\mu \mathrm{M}$. per gram per minute) was found to be quite comparable in value to that of two normal human subjects ( 1.60 and $1.95 \mu \mathrm{M}$. per gram per minute).

The behavior of the muscle esterase in this case toward the inhibiting action of prostigmine in vitro was similar to that found in the case of the guinea pig. 
Relative activity of muscle and serum choline esterase of a case of myasthenia gravis when equilibrated with known concentrations of prostigmine

Prostigmine
mgm. per liter
0.0
0.005
0.01
0.05
0.1
0.5
1.0
2.0

\begin{tabular}{lcc} 
& \multicolumn{2}{c}{ Esterase activity } \\
& Muscle & Serum \\
Therapeutic zone & 1.00 & 1.00 \\
Therapeutic zone & & 0.90 \\
Therapeutic zone & & 0.86 \\
Therapeutic zone & 1.19 & 0.57 \\
& 1.11 & 0.54 \\
& 0.73 & 0.32 \\
& 0.79 & 0.23 \\
& 0.41 &
\end{tabular}

Conclusions. 1. The inhibiting effect of prostigmine on the choline esterase of muscle and serum of guinea pigs and humans is quantitatively quite different. Only concentrations quite outside the therapeutic range depress the activity in the case of the muscle and then the effect is much less than in the case of the serum.

2. The muscle esterase content of a case of myasthenia gravis was about the same as that found in two normal human subjects.

3. Our data lend no support to the hypothesis that myasthenia gravis is associated with an unduly high choline esterase of the muscle; furthermore, the evidence is against the hypothesis that the beneficial effect of prostigmine is due to its inhibiting action upon the muscle esterase.

Clinical Study of Persons with Subnormal Temperatures.

By Hobart A. Reimann, Philadelphia, Pa.

Several years ago I collected and studied 16 patients whose temperatures were found to be slightly higher than the accepted normal. It was shown that the temperature was not true fever but was normal for these patients. Hyperthermia is rather frequently encountered in persons of a certain type, especially in women in the latter half of the menstrual cycle.

Subsequently I studied several persons, all happened to be men, whose temperature averaged below the normal level and varied between $96^{\circ} \mathrm{F}$. and $98^{\circ} \mathrm{F}$. Each of these patients came to the hospital with numerous bizarre complaints which are often associated with neurasthenia. The outstanding complaints were weakness, abdominal distress, cold and sweaty extremities, and palpitation of the heart. After complete studies were made no important deviations from the normal were found except a tendency to hypothermia, hypotension, and bradycardia. Both the blood pressure and heart rate were quickly raised by physical or emotional stress. The basal metabolic tests were usually within normal limits. Some patients reacted markedly to small doses $(1 \mathrm{mgm}$.) of pilocarpine or to $0.5 \mathrm{cc}$. doses of epinephrine solutions. Atropine, thyroid substance, opium, benzedrine, pilocarpine and epinephrine had little or no effect on the temperature. Exercise and emotional excitement sometimes caused a temporary increase in blood pressure and pulse rate.

Each of the patients can apparently be placed either in the group classed tentatively as vagotonia or sympathicotonia, but the symptoms rarely fit completely into one group or the other.

The Use of Trypsin in Producing Experimental Nephritis. By LouIs N. Katz and (by invitation) MEYER Friedman, Chicago, Ill.

An acute nephritis was produced in dogs by a single injection of a 1 per cent solution of commercial trypsin directly into both renal arteries. The nephritis was characterized by glomerular hemorrhage and inflammation, and could be made severe enough, depending upon the amount of trypsin injected, to lead to a lasting nephritis terminating in uremia. Control injections of casein and of lipase into the renal arteries led to no recognizable lesions in the kidneys or changes in their function. We are studying the effects of this trypsin nephritis upon the dogs' blood pressure.

The Effect of Sulfanilamide on Electrolyte Metabolism. By William W. Beckman (introduced by James $\mathrm{H}$. Means), Boston, Mass.

A reduction of the carbon dioxide combining power of the serum during the administration of sulfanilamide indicates that a derangement of the electrolyte metabolism takes place. In order to define better this disturbance, the acid-base excretion and serum concentration of patients receiving sulfanilamide were studied. They were kept on a constant diet and fluid intake.

The first effects noted are: a striking increase in the sodium excretion, a marked reduction in ammonia output and a strongly alkaline urine ( $\mathrm{pH} 7.4$ to 7.8 ). The serum sodium concentration falls 5 or $6 \mathrm{~m}$.eq. There occurs a corresponding diminution in the serum carbon dioxide content. Within a few days, the sodium and ammonia excretion and the urine $\mathrm{pH}$ return to pretreatment values despite continuances of the drug. The lowered serum sodium concentration, however, persists as long as sulfanilamide is given (28 days in one experiment). When the drug is discontinued the reverse phenomena are observed. Sodium is retained, ammonia excretion is increased, and the urine becomes acid. The serum sodium concentration returns to its normal level. The potassium excretion follows a course similar to the sodium but of considerably less magnitude.

In order to define more completely the changes in the internal environment of the organism caused by sulfanilamide, we are making similar observations on other electrolytes. 